\title{
Right inverses for partial differential operators on Fourier hyperfunctions
}

\author{
by \\ Michael LANGenbruch (Oldenburg)
}

Dedicated to Professor Dr. D. Vogt on the occasion of his 65th birthday

\begin{abstract}
We characterize the partial differential operators $P(D)$ admitting a continuous linear right inverse in the space of Fourier hyperfunctions by means of a dual $(\bar{\Omega})$-type estimate valid for the bounded holomorphic functions on the characteristic variety $V_{P}$ near $\mathbb{R}^{d}$. The estimate can be transferred to plurisubharmonic functions and is equivalent to a uniform (local) Phragmén-Lindelöf-type condition.
\end{abstract}

1. Introduction. Continuous linear right inverses for partial differential operators and convolution operators have been studied in many classical spaces of (generalized) functions. The problem was posed by L. Schwartz for partial differential operators in $\mathcal{C}^{\infty}\left(\mathbb{R}^{d}\right)$ and has been solved in this case and for the spaces of (ultra)distributions and ultradifferentiable functions by R. Meise, B. A. Taylor and D. Vogt (see [22, 25]) by means of a PhragménLindelöf condition. Since then corresponding results have also been obtained for weighted spaces of (ultra)distributions and (ultra)differentiable functions and convolution operators (mainly in one variable). A by no means complete list of relevant papers is contained in the references (intended as a first hint to the relevant literature, see $[1,11-14,17-23,25,28])$.

In the present paper, we will study this topic for Sato's space of Fourier hyperfunctions (see [30] and $[9,10]$ ). This space of generalized functions is the dual space $P_{*}\left(\mathbb{R}^{d}\right)^{\prime}$ of $P_{*}\left(\mathbb{R}^{d}\right):=\operatorname{limind}_{j \rightarrow \infty} P_{*, j}$, where

$$
P_{*, j}:=\left\{f \in \mathcal{H}\left(U_{j}\right)\left|\|f\|_{j}:=\sup _{z \in U_{j}}\right| f(z) \mid \exp (|z| / j)<\infty\right\}
$$

is the space of exponentially decreasing holomorphic test functions on strips

$$
U_{j}:=\left\{z \in \mathbb{C}^{d}|| \Im(z) \mid<1 / j\right\}
$$

2000 Mathematics Subject Classification: Primary 35E20; Secondary 46A63, 46F15.

Key words and phrases: partial differential operator, right inverses, Fourier hyperfunctions, Phragmén-Lindelöf conditions, $(\Omega)$-type conditions. 
near $\mathbb{R}^{d}$. We have chosen this way of defining Fourier hyperfunctions to emphasize the analogy to Schwartz' tempered distributions since $P_{*}\left(\mathbb{R}^{d}\right)$ can be considered as a holomorphic counterpart of the Schwartz space $\mathcal{S}\left(\mathbb{R}^{d}\right)$ of rapidly decreasing test functions. L. Schwartz' problem has been solved for the space $\mathcal{S}\left(\mathbb{R}^{d}\right)_{\mathrm{b}}^{\prime}$ of tempered distributions in [12] by different methods. The reader should compare the results with the present case.

Let $P(D)$ be a partial differential operator with constant coefficients in $d$ variables and let

$$
V_{P}:=\left\{z \in \mathbb{C}^{d} \mid P(-z)=0\right\}, \quad X_{P}:=V_{P} \cap \mathbb{R}^{d} .
$$

Let $d\left(x, V_{P}\right)$ and $d\left(x, X_{P}\right)$ denote the distance from $x \in \mathbb{R}^{d}$ to $V_{P}$ (and to $X_{P}$, respectively).

One might think that $P(D)$ admits a right inverse in $P_{*}\left(\mathbb{R}^{d}\right)_{\mathrm{b}}^{\prime}$ if $X_{P}=\emptyset$. While this is not true, we will prove the following characterization in this basic case: If $X_{P}=\emptyset$ then $P(D)$ admits a right inverse in $P_{*}\left(\mathbb{R}^{d}\right)_{\mathrm{b}}^{\prime}$ iff there is $\varepsilon>0$ such that $d\left(x, V_{P}\right) \geq \varepsilon$ for $x \in \mathbb{R}^{d}$ (see 2.6).

To state the characterization for $X_{P} \neq \emptyset$ we need some more notation: Let $B_{r}\left(z_{0}\right):=\left\{z \in \mathbb{C}^{d}|| z-z_{0} \mid<r\right\}$ if $z_{0} \in \mathbb{C}^{d}$, and for $f \in \mathcal{H}\left(U_{j}\right)$ let $\|f\|_{V_{P}, j}$ be the canonical norm \|\|$_{j}$ of $P_{*, j}$ restricted to $V_{P}$, i.e.

$$
\|f\|_{V_{P}, j}:=\sup _{z \in U_{j} \cap V_{P}}|f(z)| \exp (|z| / j) .
$$

Main Theorem. Let $X_{P} \neq \emptyset$. The following are equivalent:

(a) $P(D)$ admits a right inverse in $P_{*}\left(\mathbb{R}^{d}\right)_{\mathrm{b}}^{\prime}$.

(b) There is $D \in \mathbb{N}$ such that for any $j, n$ and $k$ there is $C_{0}$ such that for any $f \in \mathcal{H}\left(U_{j}\right)$,

$$
\|f\|_{V_{P}, D n j} \leq C_{0}\|f\|_{V_{P}, k}^{1-1 / n}\|f\|_{V_{P}, j}^{1 / n} .
$$

(c) There is $C_{1}>0$ such that for any $\alpha \in \mathbb{N}_{0}^{d}$,

$$
\left|P^{(\alpha)}(x)\right| \leq C_{1}|P(x)| \quad \text { if } x \in \mathbb{R}^{d} \text { and } d\left(x, X_{P}\right) \geq 1 / C_{1}
$$

and $V_{P}$ satisfies the following uniform local Phragmén-Lindelöf condition:

(UPL) loc there are $r_{1}>r_{2}>0$ and $C_{2}>0$ such that, for any $a \in X_{P}$ and any plurisubharmonic function $v$ on $V_{P} \cap B_{r_{1}}(a)$, the fact that

$$
v(x) \leq 0 \quad \text { for } x \in X_{P} \cap B_{r_{1}}(a), v(z) \leq 1 \quad \text { for } z \in V_{P} \cap B_{r_{1}}(a)
$$

implies that

Several equivalent intermediate conditions are needed in the proof of this characterization (see 3.4 and 4.6). 
The local Phragmén-Lindelöf condition $(\mathrm{PL})_{\text {loc }}(a)$ has been introduced by Hörmander in his pioneering paper [7] on surjective partial differential operators on real analytic functions. (UPL) loc is a uniform version of $(\mathrm{PL})_{\text {loc }}(a)$.

Variants of Hörmander's Phragmén-Lindelöf conditions have been intensively studied since then (see $[4,23,24,26]$ ) and were applied to several interesting problems in analysis, e.g. to characterize the surjective partial differential operators on Roumieu type ultradifferentiable functions and on real analytic functions (see $[2-5,34,35]$ ), to the right inverse problem for partial differential operators already mentioned (see $[22,25]$ ), or to characterize the real analytic varieties admitting an extension operator for real analytic functions [32]. In fact, the present paper owes very much to the point of view from [32].

The Main Theorem has several interesting implications:

If $\emptyset \neq X_{P}$ is compact then $P(D)$ admits a right inverse in $P_{*}\left(\mathbb{R}^{d}\right)_{\mathrm{b}}^{\prime}$ iff $V_{P}$ satisfies Hörmander's $(\mathrm{PL})_{\text {loc }}(a)$ for any $a \in X_{P}$.

If $P(D)$ is homogeneous then $P(D)$ admits a right inverse in $P_{*}\left(\mathbb{R}^{d}\right)_{\mathrm{b}}^{\prime}$ iff $V_{P}$ satisfies $(\mathrm{PL})_{\text {loc }}(0)$.

If $P(D)$ admits a right inverse in $P_{*}\left(\mathbb{R}^{d}\right)_{\mathrm{b}}^{\prime}$ then any localization of $P$ at $a \in X_{P}$ or at $\infty$ also admits a right inverse in $P_{*}\left(\mathbb{R}^{d}\right)_{\mathrm{b}}^{\prime}$, while the principal part $P_{m}(D)$ of $P$ in general need not.

The paper is organized as follows: In the second section, the necessity of (1.1) is shown. The proof is based on the fact that $P_{*}\left(\mathbb{R}^{d}\right)_{\mathrm{b}}^{\prime}$ is isomorphic to a power series space of finite type by Hermite expansion (see [16]). We show that $\operatorname{ker} P(D) \subset P_{*}\left(\mathbb{R}^{d}\right)_{\mathrm{b}}^{\prime}$ satisfies a strong (tame) $(\bar{\Omega})$-type condition if $P(D)$ has a right inverse in $P_{*}\left(\mathbb{R}^{d}\right)_{\mathrm{b}}^{\prime}$. By duality and a tame version of the division and extension theorem (related to the Ehrenpreis/Palamodov fundamental principle) in $P_{*}\left(\mathbb{R}^{d}\right)$ this can be translated into (1.1).

The sufficiency of (1.1) is proved in Section 3 using the tame splitting theory for exact sequences of power series spaces of finite type from [11].

In Section 4, the estimate (1.1) is transferred to psh functions using the construction of suitable holomorphic approximations for psh functions from [23]. This is used to show that (b) and (c) of the Main Theorem are equivalent.

$(\mathrm{PL})_{\text {loc }}(0)$ is equivalent to a scaled local version of (1.1) for psh functions (see 4.4(c)) which is used in Section 5 to transfer our results to several types of localizations of $P$. We also prove that $(\mathrm{PL})_{\text {loc }}(0)$ is equivalent to a local uniform radial Phragmén-Lindelöf condition combined with the following distance condition (see 4.5): there are $r_{1}>r_{2}>0$ and $B \geq 1$ such that

$$
d\left(z, X_{P} \cap B_{r_{1}}(0)\right) \leq B|\Im(z)| \quad \text { if } z \in V_{P} \cap B_{r_{2}}(0) .
$$


2. The basic tame estimates. In the following, $P(D)$ is always a partial differential operator with constant coefficients in $d$ variables and

$$
V_{P}:=\left\{z \in \mathbb{C}^{d} \mid P(-z)=0\right\} .
$$

We want to study when $P(D)$ admits a right inverse in $P_{*}\left(\mathbb{R}^{d}\right)_{\mathrm{b}}^{\prime}$, that is, when there is a continuous linear operator

$$
R: P_{*}\left(\mathbb{R}^{d}\right)_{\mathrm{b}}^{\prime} \rightarrow P_{*}\left(\mathbb{R}^{d}\right)_{\mathrm{b}}^{\prime} \quad \text { such that } P(D) \circ R=\operatorname{Id} \text { on } P_{*}\left(\mathbb{R}^{d}\right)^{\prime} .
$$

Though we will look for necessary conditions for this problem in this section, we first treat a simple sufficient condition:

ExAmPLE 2.1. $P(D)$ admits a right inverse in $P_{*}\left(\mathbb{R}^{d}\right)_{\mathrm{b}}^{\prime}$ if

$$
V_{P} \cap U_{j_{0}}=\emptyset \quad \text { for some } j_{0} \in \mathbb{N} \text {. }
$$

Proof. We first notice that there is $C>0$ such that for all polynomials $P$ with $\operatorname{deg}(P) \leq m$ we have, for any $x \in \mathbb{R}^{d}$,

$$
1 / C \leq d\left(x, V_{P}\right) \sum_{\alpha \neq 0}\left|P^{(\alpha)}(x) / P(x)\right|^{1 /|\alpha|} \leq C \quad \text { if } P(x) \neq 0
$$

(see $[8,11.1 .4])$, where $d\left(x, V_{P}\right):=\inf _{z \in V_{P}}|x-z|$ denotes the distance from $x \in \mathbb{R}^{d}$ to $V_{P}$.

Let $P_{y}(x):=P(x+i y)$ for $y \in \mathbb{R}^{d}$. Then $d\left(\cdot, V_{P_{y}}\right) \geq 1 /\left(2 j_{0}\right)$ by assumption if $|y|<1 /\left(2 j_{0}\right)$. Hence, (2.2) implies that there is $\varepsilon>0$ such that

$$
|P(z)| \geq \varepsilon \quad \text { if }|\Im(z)|<1 /\left(2 j_{0}\right),
$$

and therefore the division operator

$$
S_{\check{P}}: P_{*}\left(\mathbb{R}^{d}\right) \rightarrow P_{*}\left(\mathbb{R}^{d}\right), \quad S_{\check{P}}(f):=f / P(-\cdot),
$$

is a continuous linear operator in $P_{*}\left(\mathbb{R}^{d}\right)$. Thus, $R:=\mathcal{F} \circ S_{\check{P}}^{t} \circ \mathcal{F}^{-1}$ is a right inverse for $P(D)$ since the Fourier transformation $\mathcal{F}$ is an isomorphism in $P_{*}\left(\mathbb{R}^{d}\right)_{\mathrm{b}}^{\prime}$ (see e.g. $[6$, Chap. IV.6] or $[16,3.6])$.

Condition (2.1) means that $d\left(x, V_{P}\right) \geq 1 / j_{0}$ if $x \in \mathbb{R}^{d}$. In fact, (2.1) holds iff there is $C_{1}$ such that

$$
\left|P^{(\alpha)}(x)\right| \leq C_{1}|P(x)| \quad \text { if } \alpha \neq 0 \text { and } x \in \mathbb{R}^{d} .
$$

This can be easily checked using (2.2). We will see in 2.6 below that (2.1) is also necessary for the existence of a right inverse for $P(D)$ in $P_{*}\left(\mathbb{R}^{d}\right)_{\mathrm{b}}^{\prime}$ if $X_{P}:=V_{P} \cap \mathbb{R}^{d}=\emptyset$. From now on we will assume that

$$
V_{P} \cap U_{j} \neq \emptyset \quad \text { for any } j \text {. }
$$

In this general case, division by $\check{P}$ in $P_{*}\left(\mathbb{R}^{d}\right)^{\prime}$ needs more sophisticated methods (see Section 3 ) and we will concentrate in the rest of this section on necessary conditions for the existence of a right inverse for $P(D)$. 
We will have to use precise (so-called tame) estimates in the proofs, and recall some basic related notions first.

A Fréchet space $E$ with a fixed increasing system $\left(||_{j}\right)_{j \in \mathbb{N}}$ of seminorms defining the topology of $E$ is called a graded Fréchet space.

In this paper, $P_{*}\left(\mathbb{R}^{d}\right)_{\mathrm{b}}^{\prime}$ will always be considered with the canonical grading defined by

$$
|\nu|_{j}:=\sup \left\{|\nu(f)| \mid f \in P_{*}\left(\mathbb{R}^{d}\right),\|f\|_{j} \leq 1\right\} \quad \text { if } \nu \in P_{*}\left(\mathbb{R}^{d}\right)^{\prime} .
$$

A linear mapping

$$
T:\left(E,||_{j}\right) \rightarrow\left(F,||_{j}\right)
$$

between two graded $(\mathrm{F})$-spaces $\left(E,||_{j}\right)$ and $\left(F, \mid{ }_{j}\right)$ is called (linearly) tame if there is $A \in \mathbb{N}$ such that for any $j \in \mathbb{N}$ there is $C_{1}>0$ such that for any $f \in E$,

$$
|T(f)|_{j} \leq C_{1}|f|_{A j} .
$$

We call $T$ tame open iff there is $A \in \mathbb{N}$ such that for any $j \in \mathbb{N}$ there is $C_{1}>0$ such that for any $g \in F$ there is $f \in E$ with $T(f)=g$ such that

$$
|f|_{j} \leq C_{1}|g|_{A j}
$$

Finally, $T$ is a tame isomorphism iff $T$ is bijective, tame and tame open.

The linear topological structure of $P_{*}\left(\mathbb{R}^{d}\right)_{\mathrm{b}}^{\prime}$ is known in this precise sense by the following result from [16]:

TheOREM 2.2. $P_{*}\left(\mathbb{R}^{d}\right)_{\mathrm{b}}^{\prime}$ endowed with its canonical grading is tamely isomorphic to $\Lambda_{0}\left(k^{1 /(2 d)}\right)$ by Hermite expansion.

Recall that power series spaces of finite type and their canonical gradings are defined as follows: Let $\left(a_{k}\right)_{k \in \mathbb{N}}$ be an increasing sequence of positive numbers. Then

$$
\Lambda_{0}\left(a_{k}\right):=\left\{\left.\left(c_{k}\right)_{k \in \mathbb{N}}|\forall j \in \mathbb{N}:|\left(c_{k}\right)\right|_{j}:=\sum_{k \in \mathbb{N}}\left|c_{k}\right| e^{-a_{k} / j}<\infty\right\} .
$$

Tame maps are the appropriate tool when working with power series spaces of finite type. So we will use these precise continuity estimates in this paper, and we will always fix the grading in the $(\mathrm{F})$-spaces under consideration.

Our basic tool from the theory of partial differential equations is a tame version of the division and extension theorem in $P_{*}\left(\mathbb{R}^{d}\right)$ (see 2.3 below). Let

$$
P=P_{1} \ldots P_{r} \text { with irreducible and relatively prime factors. }
$$

Let $\varrho_{j}(f):=\left.f\right|_{V_{P} \cap U_{j}}$ and

$$
\|f\|_{V_{P}, j}:=\sup _{z \in U_{j} \cap V_{P}}|f(z)| \exp (|z| / j) \quad \text { if } f \in \mathcal{H}\left(U_{j}\right) .
$$


Theorem 2.3. Let $P$ be as in (2.5).

(a) $\varrho_{j}(P(-\cdot) f)=0$ if $f \in P_{*, j}$. Conversely, if $\varrho_{j}(f)=0$ for $f \in P_{*, j}$ then there is $g \in P_{*, 2 j}$ such that $P(-\cdot) g=f$ on $U_{2 j}$.

(b) For any $j$ there is $C_{j}$ such that for any $f \in \mathcal{H}\left(U_{j}\right)$ with $\|f\|_{V_{P}, j}<\infty$ there is $g \in P_{*, 8 j}$ such that

$$
\varrho_{8 j}(f)=\varrho_{8 j}(g), \quad\|g\|_{8 j} \leq C_{j}\|f\|_{V_{P}, j} .
$$

Proof. (a) If $\varrho_{j}(f)=0$ then $f=P(-\cdot) g$ for some $g \in \mathcal{H}\left(U_{j}\right)$ by $(2.5)$. By the Malgrange lemma (see e.g. $[8,7.3 .12]$ ) there is $C_{1}$ such that for any $z \in U_{2 j}$,

$$
|g(z)| e^{|z| /(2 j)} \leq C_{1} \sup _{|\eta| \leq 1 /(2 j)}|f(z+\eta)| e^{|z| /(2 j)} \leq C_{2}\|f\|_{j} .
$$

(b) The proof of $[8,15.3 .3]$, applied to $P_{j}(z):=P(z /(4 j))$, shows that there are $C, c_{j}$ and $C_{j}$ such that for any $F \in \mathcal{H}\left(U_{j}\right)$ there is $G \in \mathcal{H}\left(U_{4 j}\right)$ such that $\varrho_{8 j}(F)=\varrho_{8 j}(G)$ and

$$
\begin{aligned}
\sup _{z \in U_{8 j}}|G(z)| e^{-|z| /(4 j)} & \leq C\left(\int_{U_{4 j}}|G(z)|^{2} e^{-|z| /(8 j)} d z\right)^{1 / 2} \\
& \leq c_{j} C \sup _{z \in V_{P} \cap U_{j}}|F(z)| e^{-|z| /(4 j)}(1+|z|)^{c_{j}} \\
& \leq C_{j} C \sup _{z \in V_{P} \cap U_{j}}|F(z)|
\end{aligned}
$$

if the right hand side is finite. Let $f \in \mathcal{H}\left(U_{j}\right)$ with $\|f\|_{V_{P}, j}<\infty$ and set $F(z):=f(z) \prod_{l=1}^{d} \cosh \left(z_{l} / j\right)$. Then $(2.7)$ can be applied to obtain $G \in$ $\mathcal{H}\left(U_{4 j}\right)$. Set $g(z):=G(z) / \prod_{l=1}^{d} \cosh \left(z_{l} / j\right)$. For $z \in U_{8 j}$ we clearly get

$$
|g(z)| e^{|z| /(8 j)} \leq|G(z)| e^{-|z| /(4 j)} \leq C_{j} C \sup _{z \in V_{P} \cap U_{j}}|F(z)| \leq C_{j}^{\prime}\|f\|_{V_{P}, j}
$$

and $\varrho_{8 j}(f)(z)=\varrho_{8 j}(g)(z)$.

The tame invariant behind our calculations is a strong formulation of a dual $(\bar{\Omega})$-type condition. For $\left(c_{k}\right)_{k \in \mathbb{N}} \in \Lambda_{0}\left(a_{k}\right)^{\prime}$ let $\left|\left(c_{k}\right)\right|_{j}^{*}:=\sup _{k \in \mathbb{N}}\left|c_{k}\right| e^{a_{k} / j}$ be the canonical dual norms in the dual power series space $\Lambda_{0}\left(a_{k}\right)^{\prime}$ of finite type. An easy calculation shows that for any $k, n, j \in \mathbb{N}$ there is $C_{1}$ such that

$$
||_{n j}^{*} \leq C_{1}\left(||_{k}^{*}\right)^{1-1 / n}\left(||_{j}^{*}\right)^{1 / n} .
$$

Thus by 2.2 there is $B \in \mathbb{N}$ such that for any $k, n, j \in \mathbb{N}$ there is $C_{2}$ such that

$$
\|g\|_{B n j} \leq C_{2}\|g\|_{k}^{1-1 / n}\|g\|_{j}^{1 / n} \quad \text { if } g \in P_{*, j}
$$

Inequality (2.8) can be transferred to the dual of $\operatorname{ker}(P(D))$ if $P(D)$ has a right inverse in $P_{*}\left(\mathbb{R}^{d}\right)_{\mathrm{b}}^{\prime}$ and this leads to the first version of the main result 
of this section. Here and in similar subsequent estimates we set $a \infty:=\infty$ if $a \geq 0$.

TheOREM 2.4. Let $P$ satisfy (2.4) and let $P(D)$ admit a right inverse in $P_{*}\left(\mathbb{R}^{d}\right)_{\mathrm{b}}^{\prime}$. Then there is $D \in \mathbb{N}$ such that for any $j, n, k \in \mathbb{N}$ there is $C_{1}$ such that for any $f \in \mathcal{H}\left(U_{j}\right)$,

$$
\|f\|_{V_{P}, D n j} \leq C_{1}\|f\|_{V_{P}, k}^{1-1 / n}\|f\|_{V_{P}, j}^{1 / n} .
$$

Proof. (a) Let $P=P_{1}^{m_{1}} \ldots P_{r}^{m_{r}}$ with irreducible and relatively prime factors and let $R$ be a right inverse for $P(D)$ in $P_{*}\left(\mathbb{R}^{d}\right)_{\mathrm{b}}^{\prime}$. Then $R_{Q}:=$ $\prod_{i \leq r} P_{i}(D)^{m_{i}-1} R$ is a right inverse for $Q(D):=\prod_{i \leq r} P_{i}(D)$ in $P_{*}\left(\mathbb{R}^{d}\right)_{\mathrm{b}}^{\prime}$ and $V_{P}=V_{Q}$. We may thus assume that $P=Q$. We find that $R$ is tame by $[31,5.1]$ since $P_{*}\left(\mathbb{R}^{d}\right)_{\mathrm{b}}^{\prime}$ is tamely isomorphic to $\Lambda_{0}\left(k^{1 /(2 d)}\right)$ (with its canonical grading) by 2.2. Since the Fourier transformation is a tame isomorphism in $P_{*}\left(\mathbb{R}^{d}\right)_{\mathrm{b}}^{\prime}($ see $[16]), R^{t}$ defines a left inverse $L: P_{*}\left(\mathbb{R}^{d}\right) \rightarrow P_{*}\left(\mathbb{R}^{d}\right)$ for the multiplication operator $M_{\check{P}}$ in $P_{*}\left(\mathbb{R}^{d}\right)$ and there is $A \in \mathbb{N}$ such that

$$
\|L(f)\|_{A j} \leq C_{1}\|f\|_{j} \quad \text { if } f \in P_{*, j} .
$$

Let $\pi(f):=f-P(-\cdot) L(f)$ for $f \in P_{*}\left(\mathbb{R}^{d}\right)$. Then

$$
\|\pi(f)\|_{2 A j} \leq C_{2}\|f\|_{j} \quad \text { if } f \in P_{*, j} .
$$

(b) Let $f \in \mathcal{H}\left(U_{j}\right)$. By 2.3(b), for any $k \geq j$ there is $g_{k} \in P_{*, 8 k}$ such that

$$
\varrho_{8 k}\left(g_{k}\right)=\varrho_{8 k}(f), \quad\left\|g_{k}\right\|_{8 k} \leq C_{k}\|f\|_{V_{P}, k} .
$$

Notice that by $2.3(\mathrm{a})$, for any $k \geq j$,

$$
\varrho_{8 k}\left(\pi\left(g_{k}\right)\right)=\varrho_{8 k}\left(g_{k}-P(-\cdot) L\left(g_{k}\right)\right)=\varrho_{8 k}\left(g_{k}\right)=\varrho_{8 k}(f) .
$$

By 2.3(a) and (2.12) there is $h_{k} \in P_{*, 16 k}$ such that $P(-z) h_{k}(z)=g_{j}(z)-$ $g_{k}(z)$ if $z \in U_{16 k}$. This implies by the definition of $\pi$ and $(2.10)$ that $\pi\left(g_{j}\right)=$ $\pi\left(g_{k}\right)$ on $U_{17 A k}$ and hence

$$
\begin{aligned}
\|f\|_{V_{P}, 17 A B j n} & \leq\left\|\pi\left(g_{j}\right)\right\|_{17 A B j n} \leq c_{1}\left\|\pi\left(g_{j}\right)\right\|_{17 A k}^{1-1 / n}\left\|\pi\left(g_{j}\right)\right\|_{17 A j}^{1 / n} \\
& \leq c_{2}\left\|\pi\left(g_{k}\right)\right\|_{17 A k}^{1-1 / n}\left\|\pi\left(g_{j}\right)\right\|_{17 A j}^{1 / n} \leq c_{3}\left\|g_{k}\right\|_{8 k}^{1-1 / n}\left\|g_{j}\right\|_{8 j}^{1 / n} \\
& \leq c_{4}\|f\|_{V_{P}, k}^{1-1 / n}\|f\|_{V_{P}, j}^{1 / n}
\end{aligned}
$$

by (2.8) and (2.11) $-(2.13)$.

We can omit the exponential weights in (2.9) and then state (2.9) in a much stronger form. Set

$$
X_{P}:=V_{P} \cap \mathbb{R}^{d}
$$

and for $f \in \mathcal{H}\left(U_{j}\right)$ let

$$
\left\|| | f \left|\left\|_{j}:=\sup _{z \in V_{P} \cap U_{j}}|f(z)|, \quad\left|\left\|f\left|\|_{X_{P}}:=\sup _{x \in X_{P}}\right| f(x) \mid\right.\right.\right.\right.\right.
$$

$\left(\||| f \mid\|_{j}\right.$ and $\||f|\|_{X_{P}}$ may be infinite). 
Theorem 2.5. Let $V_{P}$ satisfy (2.4). Then (2.9) is valid iff $X_{P} \neq \emptyset$ and there is $D \in \mathbb{N}$ such that for any $j$ and $n$ and any $f \in \mathcal{H}\left(U_{j}\right)$,

$$
\||f|\|_{D n j} \leq\|\| f\left|\left\|_ { X _ { P } } ^ { 1 - 1 / n } \left|\|f \mid\|_{j}^{1 / n}\right.\right.\right. \text {. }
$$

Proof. Necessity. (a) (2.9) implies that for any $j, n, k \in \mathbb{N}$ and any $f \in$ $\mathcal{H}\left(U_{j}\right)$ we have

$$
\||f|\|_{D n j} \leq\|\| f\left\|_ { k } ^ { 1 - 1 / n } \left|\|f \mid\|_{j}^{1 / n} .\right.\right.
$$

Indeed, let $f \in \mathcal{H}\left(U_{j}\right)$ be bounded on $V_{P} \cap U_{j}$. Then $\|g\|_{V_{P}, j}$ is finite for $g(z):=f / \prod_{l=1}^{d} \cosh \left(z_{l} / j\right)$ and we get by (2.9), for fixed $z_{0} \in V_{P} \cap U_{D n j}$,

$$
\begin{aligned}
\left|f\left(z_{0}\right)\right| & \leq C\left(z_{0}\right)\left|g\left(z_{0}\right)\right| \leq C\left(z_{0}\right) C_{1}\|g\|_{V_{P}, k}^{1-1 / n}\|g\|_{V_{P}, j}^{1 / n} \\
& \leq C_{3}\left(z_{0}\right)\||f|\|_{k}^{1-1 / n}\|f \mid\|_{j}^{1 / n} .
\end{aligned}
$$

Applying (2.16) to $f^{m}$ instead of $f$ for $m \in \mathbb{N}$ we get (2.15).

(b) The transition to $\||f|\|_{X_{P}}$ instead of $\||f|\|_{k}$ now follows similarly to $[32,(4)]$ : When proving $(2.14)$ we may assume that \|\|$f\|\|_{D n j}>0$. Fix $z_{0} \in V_{P} \cap U_{D n j}$ such that $\||f|\|_{D n j} \leq 2\left|f\left(z_{0}\right)\right|$ and set

$$
g(z):=f(z) e^{-\left\langle z-z_{0}\right\rangle^{2}} \quad \text { where }\left\langle z-z_{0}\right\rangle^{2}:=\sum_{n \leq d} z_{j}^{2} .
$$

Choose $z_{k} \in V_{P} \cap U_{k}$ such that $\left|\|g\|_{k} \leq 2\right| g\left(z_{k}\right) \mid$. Then $\Im\left(z_{k}\right) \rightarrow 0$ and $\Re\left(z_{k}\right)$ is bounded since $g(z) \rightarrow 0$ if $\Re(z) \rightarrow \infty$ and

$$
\begin{aligned}
0 & <\||f|\|_{D n j} \leq 2\left|f\left(z_{0}\right)\right| \leq 2\left|\|g\|_{D n j} \leq 2\right|\|g\|_{k}^{1-1 / n}\|\mid\| g \|_{j}^{1 / n} \\
& \leq 4\left|g\left(z_{k}\right)\right|^{1-1 / n}\||f|\|_{j}^{1 / n} e^{4 /\left(j^{2} n\right)}
\end{aligned}
$$

since

$$
\left|e^{-\left\langle z-z_{0}\right\rangle^{2}}\right|=e^{-\left|\Re\left(z-z_{0}\right)\right|^{2}+\left|\Im\left(z-z_{0}\right)\right|^{2}} \leq e^{4 /\left(j^{2}\right)} \quad \text { if } z \in U_{j} .
$$

We can thus assume that $\zeta:=\lim z_{k}$ exists. Clearly, $\zeta \in \mathbb{R}^{d} \cap V_{P}=X_{P}$, hence this set is non-void. We may take the limit as $k \rightarrow \infty$ and obtain

$$
\begin{aligned}
\|\mid f\|_{D n j} & \leq 4 e^{4 /\left(j^{2} n\right)}|g(\zeta)|^{1-1 / n}\left\|| | \left|\left\|_{j}^{1 / n} \leq 4 e^{4 / j^{2}}|f(\zeta)|^{1-1 / n} \mid\right\| f \|_{j}^{1 / n}\right.\right. \\
& \leq 4 e^{4 / j^{2}}\left\|f \left|\left\|_{X_{P}}^{1-1 / n} \mid\right\| f \|_{j}^{1 / n} .\right.\right.
\end{aligned}
$$

As in (a), we can get rid of the constant. This proves (2.14).

Sufficiency. Let $f \in \mathcal{H}\left(U_{j}\right)$ with $\|f\|_{V_{P}, j}<\infty$. For $z_{0} \in V_{P} \cap U_{D n j}$ we set

$$
g(z):=f(z) \prod_{l=1}^{d} \cosh \left(z_{l} /(D n j)\right) e^{-\left\langle z-z_{0}\right\rangle^{2}} .
$$

Then $g$ is bounded on $V_{P} \cap U_{j}$ and

$$
\left|f\left(z_{0}\right)\right| e^{\left|z_{0}\right| /(D n j)} \leq\left|g\left(z_{0}\right)\right| \leq\|\| g\left\|_{D n j} \leq\right\||g|\left\|_{X_{P}}^{1-1 / n}\right\| \mid g \|_{j}^{1 / n}
$$


by (2.14). To pass to $f$ on the right hand side we notice that for $x \in \mathbb{R}^{d}$,

$$
\begin{aligned}
& |x| /(D n j)-\left|\Re\left(x-z_{0}\right)\right|^{2}+\left|\Im\left(z_{0}\right)\right|^{2}=|x| /(D n j)-\left|x-z_{0}\right|^{2}+2\left|\Im\left(z_{0}\right)\right|^{2} \\
& \leq C_{1}+\left|z_{0}\right| /(D n j)+\left|x-z_{0}\right| /(D n j)-\left|x-z_{0}\right|^{2} \leq C_{2}+\left|z_{0}\right| /(D n j)
\end{aligned}
$$

and therefore

$$
\||g|\|_{X_{P}}^{1-1 / n} \leq C_{3} e^{(1-1 / n)\left|z_{0}\right| /(D n j)}\|f\|_{X_{P}, k}^{1-1 / n} .
$$

For $z \in U_{j}$ we similarly get

$$
(1-1 / n)\left|z_{0}\right| /(D j)+|z| /(D n j)-\left|z-z_{0}\right|^{2}+2\left|\Im\left(z-z_{0}\right)\right|^{2} \leq C_{4}+|z| / j
$$

and therefore

$$
e^{(1-1 / n)\left|z_{0}\right| /(D j)}\left|\|g \mid\|_{j} \leq C_{5}\|f\|_{V_{P}, j} .\right.
$$

This estimate together with (2.17) implies

$$
\|f\|_{V_{P}, D n j} \leq C_{6}\|f\|_{X_{P}, k}^{1-1 / n}\|f\|_{V_{P}, j}^{1 / n} .
$$

Inequality (2.9) follows.

Combining 2.1 and 2.5 we get

Corollary 2.6. Let $X_{P}=\emptyset$. Then $P(D)$ admits a right inverse in $P_{*}\left(\mathbb{R}^{d}\right)_{\mathrm{b}}^{\prime}$ iff $V_{P} \cap U_{j_{0}}=\emptyset$ for some $j_{0}$.

A simple negative example is provided by $P(x, y):=x y+i t$ for fixed $t \in \mathbb{R}$. Clearly, $X_{P}=\emptyset$ if $t \neq 0$ and $V_{P} \cap U_{j} \neq \emptyset$ for any $j$, hence $P(D)$ admits no right inverse in $P_{*}\left(\mathbb{R}^{d}\right)_{\mathrm{b}}^{\prime}$ by 2.6. This also shows that our problem is very sensitive to small perturbations since $Q(D):=D_{1} D_{2}$ admits a right inverse in $P_{*}\left(\mathbb{R}^{d}\right)_{\mathrm{b}}^{\prime}$.

We finally show that a distance condition is valid on $V_{P}$ if $P$ satisfies (2.14). A local version is also connected to the local Phragmén-Lindelöf condition (see 4.5).

Proposition 2.7. Let $X_{P} \neq \emptyset$ and let $P$ satisfy (2.14). Then

$$
d\left(z, X_{P}\right) \leq 4 D|\Im(z)| \quad \text { if } z \in V_{P} \cap U_{2 D} .
$$

Proof. Fix $z_{0} \in V_{P} \cap U_{2 D}$ and set $g(z):=e^{-\left\langle z-z_{0}\right\rangle^{2}}$. Choose $j \in \mathbb{N}$ such that $1 /(2 D(j+1)) \leq\left|\Im\left(z_{0}\right)\right|<1 /(2 j D)$. By $(2.14)$ (for $n=2$ ) we get

$$
\begin{aligned}
1 & =g\left(z_{0}\right) \leq\|\| g\left\|_{2 D j} \leq\left|\left\|g\left|\left\|_{X_{P}}^{1 / 2}\right\| g\right|\right\|_{j}^{1 / 2} \leq e^{-d\left(z_{0}, X_{P}\right)^{2} / 2+\left|\Im\left(z_{0}\right)\right|^{2}+2 / j^{2}}\right.\right. \\
& \leq e^{-d\left(z_{0}, X_{P}\right)^{2} / 2+\left(32 D^{2}+1\right)\left|\Im\left(z_{0}\right)\right|^{2}}
\end{aligned}
$$

by the choice of $j$.

Condition (2.19) is equivalent to certain polynomial inequalities (see (2.22) below): 
REMARK 2.8. The following are equivalent:

(a) There is $A \geq 1$ such that

$$
d\left(z, X_{P}\right) \leq A|\Im(z)| \quad \text { if } z \in V_{P} \cap U_{A} .
$$

(b) There is $B \geq 1$ such that

$$
d\left(x, X_{P}\right) \leq B d\left(x, V_{P}\right) \quad \text { if } x \in \mathbb{R}^{d} \text { and } d\left(x, X_{P}\right)<1 / B .
$$

(c) There is $C \geq 1$ such that for any $\alpha$,

$$
\left|P^{(\alpha)}(x)\right| \min \left\{1 / C, d\left(x, X_{P}\right)\right\}^{|\alpha|} \leq C|P(x)| \quad \text { if } x \in \mathbb{R}^{d} .
$$

Proof. (a) $\Rightarrow$ (b). For $x \in \mathbb{R}^{d}$ with $d\left(x, X_{P}\right)<1 / A$ we choose $z \in V_{P}$ such that $|x-z|=d\left(x, V_{P}\right)$. Then

$$
|\Im(z)| \leq d\left(x, V_{P}\right) \leq d\left(x, X_{P}\right)<1 / A .
$$

Choose $y \in X_{P}$ such that $|z-y|=d\left(z, X_{P}\right)$. Then (2.20) implies

$$
\begin{aligned}
d\left(x, X_{P}\right) & \leq|x-y| \leq|x-z|+|z-y| \leq d\left(x, V_{P}\right)+d\left(z, X_{P}\right) \\
& \leq d\left(x, V_{P}\right)+A|\Im(z)| \leq(A+1) d\left(x, V_{P}\right) .
\end{aligned}
$$

(b) $\Rightarrow$ (c). If $d\left(x, X_{P}\right)<1 / B$ we get, by (b) and (2.2),

$$
d\left(x, X_{P}\right) \sum_{\alpha \neq 0}\left|P^{(\alpha)}(x) / P(x)\right|^{1 /|\alpha|} \leq B d\left(x, V_{P}\right) \sum_{\alpha \neq 0}\left|P^{(\alpha)}(x) / P(x)\right|^{1 /|\alpha|} \leq B C_{1}
$$

and this implies (2.22) for these $x$. If $d\left(x, X_{P}\right) \geq 1 / B$ then (2.2) shows that

$$
\left|P^{(\alpha)}(x)\right|(1 / B)^{|\alpha|} \leq C_{1}^{|\alpha|}|P(x)|
$$

if $\alpha \neq 0$. This completes the proof of (2.22).

(c) $\Rightarrow(\mathrm{a})$. By $(2.22)$ and (2.2) there is $C_{1}$ such that

$$
\min \left\{1 / C, d\left(x, X_{P}\right)\right\} \leq C_{1} d\left(x, V_{P}\right) \quad \text { if } x \in \mathbb{R}^{d} .
$$

Let $z \in V_{P} \cap U_{C C_{1}}$. Then $d\left(\Re(z), X_{P}\right) \leq 1 / C$ since otherwise (2.23) implies that

$$
1 / C \leq C_{1} d\left(\Re(z), V_{P}\right) \leq C_{1}|\Im(z)|<1 / C,
$$

a contradiction. Choose $y \in X_{P}$ such that $|y-\Re(z)|=d\left(\Re(z), X_{P}\right)$. Then

$$
\begin{aligned}
d\left(z, X_{P}\right) & \leq|z-y| \leq d\left(\Re(z), X_{P}\right)+|\Im(z)| \leq C_{1} d\left(\Re(z), V_{P}\right)+|\Im(z)| \\
& \leq\left(C_{1}+1\right)|\Im(z)|
\end{aligned}
$$

by $(2.23)$ since $d\left(\Re(z), X_{P}\right) \leq 1 / C$.

3. Tame splitting theory. We will show in this section that the estimates (2.9) and (2.14) are also sufficient for the existence of a right inverse for $P(D)$ in $P_{*}\left(\mathbb{R}^{d}\right)_{\mathrm{b}}^{\prime}$. We will use tame splitting theory to solve this problem; we recall the corresponding basic notions and facts first. An exact sequence

$$
0 \rightarrow E \stackrel{S}{\longrightarrow} F \stackrel{T}{\longrightarrow} G \rightarrow 0
$$


of graded (F)-spaces $E, F$ and $G$ is called tame exact iff $S$ is a tame isomorphism onto its range (with the grading induced from $F$ ) and $T$ is tame and tame open. The splitting of (3.1) is decided by means of the tame invariants $(\underline{\mathrm{DN}})_{t}$ and $(\bar{\Omega})_{t}$ which are defined as follows (see e.g. [11, 1.3]): a graded (F)-space $\left(E,||_{j}\right)$ satisfies $(\underline{\mathrm{DN}})_{t}$ if there are $A \in \mathbb{N}$ and $C \geq 1$ such that for any $n \geq C$ there are $j$ and $C_{1}$ such that

$$
\left.\left|{ }_{n} \leq C_{1}\right|\right|_{A} ^{1 /(C n)}||_{j}^{1-1 /(C n)} .
$$

A space $E$ satisfies $(\bar{\Omega})_{t}$ if for any $j$ there is $j \leq D \in \mathbb{N}$ such that for any $n \geq D$ and $k$ there is $C_{1}$ such that for any $t>0$,

$$
W_{n D} \subset t^{1 / n} W_{k}+\frac{C_{1}}{t^{1-1 / n}} W_{j},
$$

where $W_{l}:=\left\{\left.f \in E|| f\right|_{l}<1\right\}$ is the unit ball with respect to ||$_{l}$.

We will be checking $(\bar{\Omega})_{t}$ in a dual formulation. In fact, $E$ satisfies $(\bar{\Omega})_{t}$ iff for any $j$ there is $j \leq D \in \mathbb{N}$ such that for any $n \geq D$ and $k$ there is $C_{1}$ such that

$$
|y|_{D n}^{*} \leq C_{1}\left(|y|_{k}^{*}\right)^{1-1 / n}\left(|y|_{j}^{*}\right)^{1 / n} \quad \text { if } y \in E^{\prime},
$$

where $|y|_{l}^{*}:=\sup _{|f|_{l} \leq 1}|y(f)|$ are the dual seminorms for ||$_{l}$ (see e.g. [33, 1.9] and compare (2.8)).

Notice that $(\underline{\mathrm{DN}})_{t}$ and $(\bar{\Omega})_{t}$ are inherited by tame isomorphisms and that any power series space of finite type satisfies $(\underline{\mathrm{DN}})_{t}$ and $(\bar{\Omega})_{t}$ (see $[11,1.4(\mathrm{~b})])$. The following tame splitting theorem (see $[11,1.6])$ is a special case of the general splitting theorem from [29].

Theorem 3.1. Let E, $F$ and $G$ be graded (FN)-spaces and let

$$
0 \rightarrow E \stackrel{S}{\longrightarrow} F \stackrel{T}{\longrightarrow} G \rightarrow 0
$$

be a tame exact sequence. Then the sequence is split if $E$ and $G$ satisfy $(\underline{\mathrm{DN}})_{t}$ and $(\bar{\Omega})_{t}$.

We want to apply 3.1 to the sequence

$$
0 \rightarrow \operatorname{ker}(P(D)) \stackrel{\mathrm{Id}}{\longrightarrow} P_{*}\left(\mathbb{R}^{d}\right)_{\mathrm{b}}^{\prime} \stackrel{P(D)}{\longrightarrow} P_{*}\left(\mathbb{R}^{d}\right)_{\mathrm{b}}^{\prime} \rightarrow 0,
$$

which is exact by [19, Remark after 3.3]. Notice that many of the assumptions of 3.1 are satisfied for (3.4) for any $P(D)$. Since $\operatorname{ker}(P(D))$ is endowed with the grading induced by $P_{*}\left(\mathbb{R}^{d}\right)_{\mathrm{b}}^{\prime}$, the identity is a tame isomorphism. Clearly, $P(D)$ is also tame. $P_{*}\left(\mathbb{R}^{d}\right)_{\mathrm{b}}^{\prime}$ satisfies $(\underline{\mathrm{DN}})_{t}$ and $(\bar{\Omega})_{t}$ by 2.2 and (3.3), and $\operatorname{ker}(P(D))$ satisfies $(\underline{\mathrm{DN}})_{t}$ since $(\underline{\mathrm{DN}})_{t}$ is inherited by subspaces. Hence 3.1 applies if $\operatorname{ker}(P(D))$ satisfies $(\bar{\Omega})_{t}$ and if $P(D)$ is tame open. This is shown in the next two lemmata.

To check $(\bar{\Omega})_{t}$ we will prove (3.2) for the dual space of $\operatorname{ker}(P(D))$, which may be identified with the quotient space $P_{*}\left(\mathbb{R}^{d}\right) / P(-\cdot) P_{*}\left(\mathbb{R}^{d}\right)$ via Fourier 
transformation since the multiplication operator $M_{\check{P}}$ is surjective and since $P_{*}\left(\mathbb{R}^{d}\right)_{\mathrm{b}}^{\prime}$ is an (FS)-space.

Lemma 3.2. Let $X_{P} \neq \emptyset$ and let $P$ satisfy (2.5) and (2.14). Then $\operatorname{ker}(P(D))$ satisfies $(\bar{\Omega})_{t}$.

Proof. $\operatorname{ker}(P(D))$ is tamely isomorphic to $E:=\operatorname{ker}\left(M_{\check{P}}\right)$ by Fourier transformation. Recall that the grading on $E \subset P_{*}\left(\mathbb{R}^{d}\right)_{\mathrm{b}}^{\prime}$ is defined by

$$
|\nu|_{j}:=\sup \left\{|\nu(f)| \mid f \in P_{*}\left(\mathbb{R}^{d}\right),\|f\|_{j} \leq 1\right\} \quad \text { if } \nu \in E .
$$

Let $[f] \in E^{\prime}=P_{*}\left(\mathbb{R}^{d}\right) / P(-\cdot) P_{*}\left(\mathbb{R}^{d}\right)$. To show (3.2) we fix $j$ and we may assume that $|[f]|_{j}^{*}<\infty$. Then there is $g \in P_{*, j}$ such that

$$
g \in[f] \text { and }\|g\|_{V_{P}, 2 j} \leq\|g\|_{2 j} \leq|[f]|_{j}^{*}<\infty .
$$

Indeed,

$$
|\nu(f)|=|[f](\nu)| \leq|[f]|_{j}^{*}|\nu|_{j} \quad \text { if } \nu \in E=\operatorname{ker}\left(M_{\check{P}}\right) .
$$

By the Hahn-Banach theorem there is $g \in P_{*}\left(\mathbb{R}^{d}\right)$ such that $\nu(g)=\nu(f)$ if $\nu \in E$ (hence $g \in[f]$ ) and such that

$$
|\mu(g)| \leq|[f]|_{j}^{*}|\mu|_{j} \quad \text { if } \mu \in P_{*}\left(\mathbb{R}^{d}\right)^{\prime} .
$$

For $z:=x+i y \in U_{2 j}$ we apply this estimate to

$$
\mu_{z, m}:=\exp (|z| / j) \sum_{l \leq m} \delta_{x}^{(l)} / l ! \in P_{*}\left(\mathbb{R}^{d}\right)^{\prime}
$$

and conclude that $g \in P_{*, 2 j}$ and that $g$ satisfies (3.5). Moreover, for fixed $k$,

$$
\|g\|_{X_{P}, 2 k} \leq|[f]|_{k}^{*}<\infty .
$$

Indeed, as above we get $\widetilde{g} \in[f]$ such that

$$
|[f]|_{k}^{*} \geq\|\widetilde{g}\|_{V_{P}, 2 k} \geq\|\widetilde{g}\|_{X_{P}, 2 k}=\|g\|_{X_{P}, 2 k}
$$

since $g, \widetilde{g} \in[f]$, hence $\widetilde{g}=g$ on $V_{P} \cap U_{l}$ for some $l$ and therefore $\widetilde{g}=g$ on $X_{P}=V_{P} \cap \mathbb{R}^{d}$.

By (3.5), (3.6) and (2.18) we get

$$
\left(|[f]|_{j}^{*}\right)^{1 / n}\left(|[f]|_{k}^{*}\right)^{1-1 / n} \geq\|g\|_{V_{P}, 2 j}^{1 / n}\|g\|_{X_{P}, 2 k}^{1-1 / n} \geq C_{1}\|g\|_{V_{P}, 2 D n j} \geq C_{2}|[f]|_{16 D n j}^{*} .
$$

The last estimate is seen as follows: By 2.3(b) there is $\widetilde{g} \in P_{*, 16 D n j}$ such that $g=\tilde{g}$ on $V_{P} \cap U_{16 D n j}$ (hence $[f]=[g]=[\widetilde{g}]$ ) and $\|\widetilde{g}\|_{16 D n j} \leq C_{3}\|g\|_{V_{P}, 2 D n j}$. This completes the proof, since $|[f]|_{16 D n j}^{*}=|[\widetilde{g}]|_{16 D n j}^{*} \leq\|\widetilde{g}\|_{16 D n j}$ by definition.

Lemma 3.3. $P(D): P_{*}\left(\mathbb{R}^{d}\right)_{\mathrm{b}}^{\prime} \rightarrow P_{*}\left(\mathbb{R}^{d}\right)_{\mathrm{b}}^{\prime}$ is tame open if $P$ satisfies $(2.5)$ and (2.14).

Proof. (a) Clearly,

$$
M_{l}:=M_{\check{P}}: P_{*, l} \rightarrow P_{*, 2 l}
$$


is continuous. Let $\mathcal{K}_{l}:=\operatorname{ker}\left(M_{l}^{t}\right) \subset\left(P_{*, 2 l}\right)^{\prime}$. We then have the following density result: $\operatorname{ker}\left(M_{\breve{P}}\right)$ is dense in $\mathcal{K}_{8 D j}$ with respect to $\mid{ }_{j}$ for any $j$. Indeed, ||$_{j}$ is the canonical (dual) norm on $P_{*, j}^{\prime}$. Let $g \in\left(P_{*, j}^{\prime}\right)^{\prime}$ be such that $g\left(\operatorname{ker}\left(M_{\check{P}}\right)\right)=0$. Passing by the reflexive spaces $\widetilde{P}_{*, l}$ defined with $L_{2^{-}}$ norms instead of sup-norms we see that $g \in P_{*, 2 j}$. Since $\delta_{x} \in \operatorname{ker}\left(M_{\check{P}}\right)$ if $x \in X_{P}$, we know that $g\left(X_{P}\right)=0$ and therefore $g\left(V_{P} \cap U_{4 D j}\right)=0$ by $(2.14)$ for $n=2$. This implies by 2.3(a) that $g=P(-\cdot) h$ for some $h \in P_{*, 8 D j}$ and therefore

$$
\langle g, \nu\rangle=\langle P(-\cdot) h, \nu\rangle=0
$$

if $\nu \in \mathcal{K}_{8 D j}$.

(b) To prove the lemma, it is sufficient to show that

$$
M_{\check{P}}: P_{*}\left(\mathbb{R}^{d}\right)_{\mathrm{b}}^{\prime} \rightarrow P_{*}\left(\mathbb{R}^{d}\right)_{\mathrm{b}}^{\prime} \text { is tame open. }
$$

Let $\nu \in P_{*}\left(\mathbb{R}^{d}\right)^{\prime}$ with $|\nu|_{2 j} \leq 1$. For $k \geq j$ let

$$
H_{k}: E_{k}:=P(-\cdot) P_{*, k} \rightarrow \mathbb{C}, \quad H_{k}(f):=\nu(g) \quad \text { if } f=P(-\cdot) g, g \in P_{*, k} .
$$

By (2.6) we have

$$
\left|H_{k}(f)\right|=|\nu(g)| \leq|\nu|_{2 k}\|g\|_{2 k} \leq|\nu|_{2 k}\|f\|_{k} .
$$

By the Hahn-Banach theorem, $H_{k}$ may be extended to $\mu_{k} \in\left(P_{*, k}\right)^{\prime}$ such that

$$
\left|\mu_{k}\right|_{k} \leq|\nu|_{2 k}
$$

Clearly, $\left\langle M_{\breve{P}} \mu_{2 k}, f\right\rangle=\nu(f)$ if $f \in P_{*, k}$. If $k \geq l \geq j$ then $\left(\mu_{2 k}-\mu_{2 l}\right)\left(E_{l}\right)=0$ and hence $\left(\mu_{2 k}-\mu_{2 l}\right) \in \mathcal{K}_{l}$. Since $\operatorname{ker}\left(M_{\check{P}}\right)$ is dense in $\mathcal{K}_{8 D l}$ with respect to ||$_{l}$ for any $l$ by (a), we may use the classical Mittag-Leffler argument to get $\mu \in P_{*}\left(\mathbb{R}^{d}\right)^{\prime}$ such that $P(-\cdot) \mu=\nu$ and $|\mu|_{j} \leq 2$ by (3.8).

TheOrem 3.4. Let $X_{P} \neq \emptyset$. The following are equivalent:

(a) $P(D)$ admits a right inverse in $P_{*}\left(\mathbb{R}^{d}\right)_{\mathrm{b}}^{\prime}$.

(b) There is $D$ such that for any $j, n$ and $k$ there is $C_{1}$ such that for any $f \in \mathcal{H}\left(U_{j}\right)$,

$$
\|f\|_{V_{P}, D n j} \leq C_{1}\|f\|_{V_{P}, k}^{1-1 / n}\|f\|_{V_{P}, j}^{1 / n} .
$$

(c) There is $D \in \mathbb{N}$ such that for any $j$ and $n$ and any $f \in \mathcal{H}\left(U_{j}\right)$,

$$
\||f|\|_{D n j} \leq\||f|\|_{X_{P}}^{1-1 / n}\left|\|f \mid\|_{j}^{1 / n} .\right.
$$

Proof. By 2.4 and 2.5 we only have to show (c) $\Rightarrow$ (a). We may assume that $P$ is as in (2.5) since then any prime factor of $P$ and therefore also $P(D)$ has a right inverse. The claim now follows from 3.1 (and the remarks following that theorem) by 3.2 and 3.3 . 
4. Uniform Phragmén-Lindelöf conditions. Having an estimate for holomorphic functions on an algebraic variety $V$, one of the main goals is to transfer the estimate to plurisubharmonic (psh) functions on $V$ since these have more flexibility than holomorphic functions in concrete constructions. This will lead to a uniform condition of Phragmén-Lindelöf type and to the corresponding local version, connecting our problem to the manifold results which have been obtained for such conditions in recent times (see $[4,5,7$, $22-26,32,34,35])$.

The following theorem transfers the crucial condition (2.14) from 2.5 to psh functions defined on $V_{P} \cap U_{j}$. Recall that a function $u \neq-\infty$ is (weakly) psh on an algebraic variety $V$ (in symbols $u \in \mathcal{P S H}(V)$ ) if $u$ is locally bounded from above and upper semicontinuous (usc) on $V$ and if $u$ is psh near the regular points.

For $u \in \mathcal{P} \mathcal{S H}\left(V_{P} \cap U_{j}\right)$ we set, just as before,

$$
\|u \mid\|_{V_{P}, j}:=\|u\| \|_{j}:=\sup _{z \in V_{P} \cap U_{j}} u(z) \text { and }\|\mid u\|_{X_{P}}:=\sup _{x \in X_{P}} u(x) .
$$

Theorem 4.1. Let $X_{P} \neq \emptyset$ and let $P$ satisfy (2.14). For any $j, n \in \mathbb{N}$ and any $v \in \mathcal{P} \mathcal{S H}\left(V_{P} \cap U_{j}\right)$ we have, with $D$ from (2.14),

$$
\|v\|_{4 D n j} \leq\left(1-\frac{1}{n}\right)\|\| v\left\|_{X_{P}}+\frac{1}{n} \mid\right\| v \|_{j} \quad \text { if }\|v\| \|_{j}<\infty .
$$

Proof. The main step is the proof of the following claim (for $D$ from (2.14)): For any $0<\theta<1$, any $n, k, j \in \mathbb{N}$ with $k>j$ and any $z_{0} \in$ $V_{P} \cap U_{4 D n j}$ there is $C_{1}$ such that for any $v \in \mathcal{P} \mathcal{S H}\left(V_{P} \cap U_{j}\right)$ satisfying

$$
v(z) \geq 1 \quad \text { if } z \in V_{P} \cap U_{j}
$$

we have

$$
v\left(z_{0}\right) \leq C_{1}+\frac{1}{\theta}\left(1-\frac{1}{n}\right)\|v\|_{k}+\left(\frac{1}{\theta n}+1-\theta\right)\|\| v \|_{j} .
$$

We first show that the claim implies (4.1): we may assume that $v \neq-\infty$ on $V_{P} \cap U_{4 D n j}$ and that $\|v \mid\|_{j}<\infty$. Set

$$
v_{c, t}(z):=\max \{v(z)+c+1,1\} t, \quad z \in V_{P} \cap U_{j},
$$

for $c>0$ and $t \geq 1$. Clearly, $v_{c, t} \geq 1$. Let $z_{0} \in V_{P} \cap U_{4 D n j}$. If $v\left(z_{0}\right)>-\infty$ then we choose $c>0$ such that $v\left(z_{0}\right) \geq-c$ and get by (4.3), for $t \geq 1$,

$$
\begin{aligned}
t & \leq t\left(v\left(z_{0}\right)+c+1\right)=v_{c, t}\left(z_{0}\right) \\
& \leq C_{1}+\frac{1}{\theta}\left(1-\frac{1}{n}\right)\|\| v_{c, t}\left\|_{k}+\left(\frac{1}{\theta n}+1-\theta\right)\right\|\left\|v_{c, t}\right\|_{j} \\
& =C_{1}+\frac{t}{\theta}\left(1-\frac{1}{n}\right)\left\|\widetilde{v}_{c}\right\|\left\|_{k}+t\left(\frac{1}{\theta n}+1-\theta\right)\right\| v \|_{j}+(c+1) t\left(\frac{1}{\theta}+1-\theta\right)
\end{aligned}
$$


for $\widetilde{v}_{c}:=\max \{v(z),-c\}$ since $\left\|v_{c, t}\right\|_{k}=t\left(\left\|\left|\widetilde{v}_{c} \|\right|_{k}+c+1\right)\right.$ and $\left\|v_{c, t}\right\|_{j}=$ $t\left(\|\| v\|\|_{j}+c+1\right)$ since $z_{0} \in V_{P} \cap U_{4 D n j} \subset V_{P} \cap U_{j}$. Dividing by $t$ and letting $t \rightarrow \infty$ we get

$$
v\left(z_{0}\right) \leq \frac{1}{\theta}\left(1-\frac{1}{n}\right)\left\|\widetilde{v}_{c}\right\|_{k}+\left(\frac{1}{\theta n}+1-\theta\right)\|\| v \|_{j}+(c+1)\left(\frac{1}{\theta}-\theta\right) .
$$

Letting $\theta \uparrow 1$ we get

$$
v\left(z_{0}\right) \leq\left(1-\frac{1}{n}\right)\left\|\widetilde{v}_{c}\right\|_{k}+\frac{1}{n}\|v\|_{j} \quad \text { if } v\left(z_{0}\right)>-\infty .
$$

Hence $v \neq-\infty$ on $V_{P} \cap U_{k}$ since $v \neq-\infty$ on $V_{P} \cap U_{4 D n j}$ and therefore (as $c \rightarrow \infty)$

$$
\left\|\left|\left\|_{4 D n j} \leq\left(1-\frac{1}{n}\right)\left|\|v\|_{k}+\frac{1}{n}\right|\right\| v \|_{j} .\right.\right.
$$

We may replace \|\|$v\|\|_{k}$ in (4.4) by $\|v \mid\|_{X_{P}}$ as in the proof of 2.5. Notice that $\limsup _{k \rightarrow \infty} v\left(z_{k}\right) \leq v\left(\lim _{k \rightarrow \infty} z_{k}\right)$ since $v$ is psh. We thus obtain

$$
\left\|v\left|\left\|_{4 D n j} \leq C_{1}^{\prime}+\left(1-\frac{1}{n}\right)\right\|\right| v\right\|_{X_{P}}+\frac{1}{n}\|\mid v\|_{j} .
$$

As above, we can get rid of the constant $C_{1}^{\prime}$. This proves (4.1).

Let $1 \leq v \in \mathcal{P S H}\left(V_{P} \cap U_{j}\right)$ be bounded from above and let $\kappa:=\|v \mid\|_{j}+1$. Let $z_{0} \in V_{P} \cap U_{4 D n j}$. We first construct a kind of local extension $u \in$ $\mathcal{P S H}\left(V_{P}\right)$ of $v$ as follows: Let $\vartheta(z):=(|\Im(z)|-1 / k) \kappa j k /(k-j)$ if $|\Im(z)| \geq$ $1 / k$ and $\vartheta(z):=0$ if $|\Im(z)| \leq 1 / k$. Then $\vartheta$ is psh on $\mathbb{C}^{d}$. For $z \in V_{P}$ let $\widetilde{u}(z):=\max \{v(z), \vartheta(z)\}$ if $|\Im(z)| \leq 1 / j$ and $\widetilde{u}(z):=\vartheta(z)$ if $|\Im(z)| \geq 1 / j$. Then $\widetilde{u} \in \mathcal{P} \mathcal{S H}\left(V_{P}\right)$ by the definition of $\kappa$ and

$$
\|\widetilde{u}\|_{k}=\||v|\|_{k} \quad \text { and } \quad\|\widetilde{u}\|_{j} \leq\||v|\|_{j}+1 \text {. }
$$

Set $u(z):=\widetilde{u}(z)-\left|\Re\left(z-z_{0}\right)\right|^{2}+\left|\Im\left(z-z_{0}\right)\right|^{2}$ if $z \in V_{P}$. If we prove that

$$
u\left(z_{0}\right) \leq C_{2}+\frac{1}{\theta}\left(1-\frac{1}{n}\right)\|\widetilde{u}\|_{k}+\left(\frac{1}{\theta n}+1-\theta\right)\|\widetilde{u}\|_{j}
$$

then (4.3) follows since

$$
\begin{aligned}
v\left(z_{0}\right) & \leq u\left(z_{0}\right) \leq C_{2}+\frac{1}{\theta}\left(1-\frac{1}{n}\right)\|\widetilde{u}\|_{k}+\left(\frac{1}{\theta n}+1-\theta\right)\|\widetilde{u}\|_{j} \\
& \leq C_{3}+\frac{1}{\theta}\left(1-\frac{1}{n}\right)\|v\|\left\|_{k}+\left(\frac{1}{\theta n}+1-\theta\right)\right\| v \mid \|_{j}
\end{aligned}
$$

by (4.6) and (4.5).

To prove (4.6) we need a set of holomorphic functions $f_{z}$ for $z \in V_{P}$ near $z_{0}$ such that $f_{z}(z)$ almost equals $u(z)$ and such that $f_{z}$ essentially has the same growth as $u$ on $V_{P}$ so that we can then apply (2.14). A possible construction of such functions was explained in [23] and a modification will 
suit our purposes. We will use the notation from [23] and refer to that paper for any details. We may assume that $P$ satisfies $(2.5)$ and that the first unit vector is non-characteristic for $P$. Then the discriminant $D(w)$ for $w \in \mathbb{C}^{d-1}$ does not vanish identically. The set

$$
S_{0}:=\left\{(s, w) \in \mathbb{C} \times \mathbb{C}^{d-1}|| D(w) \mid<\delta(1+|w|)^{-C}\right\}
$$

contains the singular points of $V_{P}$. Using $D(w)$ we can define a pseudoconvex open neighborhood $\Omega$ of the regular points with the properties listed in $[23$, 4.1] and such that for any psh $u$ on a neighborhood of $\overline{V_{P} \cap S_{0}}$ we have

$$
u(z) \leq \max \left\{u(\zeta)\left|\zeta \in V_{P} \backslash S_{0},\right| \zeta-z \mid \leq 1 /(12 k)\right\}
$$

(cf. [23, 4.1(iv)]). In fact we may use the construction of $[23,4.1]$ for $P(12 k z)$ instead of $P$ and then shrink the coordinates again to arrive at (4.7).

To prove (4.6) for $z_{0} \in V_{P} \cap U_{4 D n j}$ we may assume that $z_{0} \in\left(V_{P} \backslash S_{0}\right) \cap$ $U_{3 n D j}$ by (4.7) if $k>n j$ (without loss of generality). By [23, 4.1] we then have $z_{0}=\left(s_{i}(w), w\right)$, where $s_{i}(w+\cdot)$ is a holomorphic function on $B:=$ $\left\{\tau \in \mathbb{C}^{d-1}|| \tau \mid<\varepsilon\left(z_{0}\right)\right\}$ such that $\left.z(\tau):=\left(s_{i}(\tau+w), \tau+w\right)\right) \in V_{P} \cap \Omega$ and the radius satisfies $\varepsilon\left(z_{0}\right)=\varepsilon_{1}\left(1+\left|z_{0}\right|\right)^{-C_{1}}$ and

$$
\left|z(\tau)-z_{0}\right| \leq 1 /(12 k) \quad \text { if }|\tau| \leq 8 \varepsilon\left(z_{0}\right)
$$

(by the argument after (4.7), cf. also $[23,4.1(\mathrm{v})(\mathrm{c})]$ ).

With a constant $C$ to be determined later we now define holomorphic functions $f_{\tau}$ on $\Omega$ as in the proof of $[23,5.1]$ (using $[23,3.2]$ with $\psi$ defined by $[23,(5.5)])$ for $\delta:=C \varepsilon\left(z_{0}\right) /\left(\left(1+\left|z_{0}\right|\right) \kappa\right)$. Notice that

$$
u(z) \geq v(z)-\left|z-z_{0}\right|^{2} \geq 0 \quad \text { if }\left|z-z_{0}\right| \leq 1 .
$$

By the proof of $[23,5.1]$ (see $[23,(5.7)]$ ) there is an exceptional set $E \subset B$ such that for $\tau \in B \backslash E$ we have, for $r$ defined by $(1-r) /(1+r)=\theta$,

$$
\begin{aligned}
\ln \left|f_{\tau}(z(\tau))\right| & \geq \theta u(z(\tau))+\ln (r \delta / 2)-C_{4} \ln (2+|z(\tau)|) \\
& \geq \theta u(z(\tau))-\ln \kappa-C_{5} \ln \left(2+\left|z_{0}\right|\right) \\
& \geq \theta u(z(\tau))-(1-\theta) \theta(\kappa-1) / 2-C_{6} \ln \left(2+\left|z_{0}\right|\right) \\
& \geq \theta u(z(\tau))-(1-\theta) \theta|| \widetilde{u} \mid \|_{j} / 2-C_{7}\left(z_{0}\right)
\end{aligned}
$$

by the definition of $\kappa$ and $\widetilde{u}$.

The measure of the exceptional set $E$ is estimated in [23, p. 304] by

$$
\begin{aligned}
|E| \leq & C_{8}|B| \frac{\delta}{\varepsilon\left(z_{0}\right)} \sup _{|\tau| \leq 2 \varepsilon\left(z_{0}\right)}\left\{u\left(s_{i}(w+\tau), w+\tau\right)\right. \\
& \left.+C_{9}(\ln |D(w)|+\ln (2+|w|))\right\} \\
\leq & C C_{8}|B|\left(|||u| \|_{j}+C_{10} \ln (2+|w|)\right) /\left(\kappa\left(1+\left|z_{0}\right|\right)\right)
\end{aligned}
$$




$$
\begin{aligned}
& \leq C C_{8}|B|\left(\kappa+C_{11} \ln \left(2+\left|z_{0}\right|\right)\right) /\left(\kappa\left(1+\left|z_{0}\right|\right)\right) \\
& \leq C C_{8}\left(1+2 C_{11}\right)|B| \leq|B|(1-\theta) / 2
\end{aligned}
$$

if $C=(1-\theta) /\left(2 C_{8}\left(1+2 C_{11}\right)\right)$, where we have used (4.5) and the fact that $\||u|\|_{j} \leq\|\mid\| \widetilde{u} \|_{j}+1 \leq \kappa+1$.

We thus deduce by the subaveraging property of psh functions, (4.9) and (4.10) that

$$
\begin{aligned}
u\left(z_{0}\right) & \leq \frac{1}{|B|} \int_{B} u(z(\tau)) d \tau=\frac{1}{|B|} \int_{E} u(z(\tau)) d \tau+\frac{1}{|B|} \int_{B \backslash E} u(z(\tau)) d \tau \\
& \leq \frac{|E|}{|B|}\left|\left\|u\left|\|_{j}+2 C_{7}\left(z_{0}\right)+\frac{(1-\theta)|\|| \widetilde{u}||_{j}}{2}+\frac{1}{\theta} \sup _{\tau \in B \backslash E} \ln \right| f_{\tau}(z(\tau)) \mid\right.\right. \\
& \leq C_{12}+(1-\theta)\left|\|\widetilde{u}\|_{j}+\frac{1}{\theta} \sup _{\tau \in B \backslash E} \ln \right| f_{\tau}(z(\tau)) \mid .
\end{aligned}
$$

By (2.14) and [23, 3.2(iii)], for $\tau \in B \backslash E$, and hence $z(\tau) \in V_{P} \cap U_{2 D n j}$ since $z_{0} \in U_{3 D n j}$, we get

$$
\begin{aligned}
& \ln \left|f_{\tau}(z(\tau))\right| \leq(1-1 / n)|||\ln | f_{\tau}||||_{X_{P}}+|||\ln | f_{\tau}||||_{2 j} / n \\
& \leq(1-1 / n)\left\|\left|u+C_{13} \ln (2+|\cdot|)\right|\right\|_{k}+\left\|u+C_{13} \ln (2+|\cdot|)\right\| \|_{j} / n \\
& =\left.(1-1 / n)|||\widetilde{u}-| \Re\left(\cdot-z_{0}\right)\right|^{2}+\left|\Im\left(\cdot-z_{0}\right)\right|^{2}+C_{13} \ln (2+|\cdot|) \mid \|_{k} \\
& +\left.|||\widetilde{u}-| \Re\left(\cdot-z_{0}\right)\right|^{2}+\left|\Im\left(\cdot-z_{0}\right)\right|^{2}+C_{13} \ln (2+|\cdot|) \mid \|_{j} / n \\
& \leq C_{14}\left(z_{0}\right)+(1-1 / n)\|\| \widetilde{u}\left\|_{k}+\right\| \widetilde{u} \|_{j} / n \text {. }
\end{aligned}
$$

Combining this estimate with (4.11) we get

$$
u\left(z_{0}\right) \leq C_{15}+(1-1 / n)\|\| \widetilde{u}\left\|_{k} / \theta+(1 /(n \theta)+1-\theta)\right\|\|\widetilde{u}\|_{j}
$$

This completes the proof of (4.6) and of Theorem 4.1.

Inequality (4.1) implies a uniform Phragmén-Lindelöf condition on $V_{P}$ near $\mathbb{R}^{d}$ :

Proposition 4.2. Let $X_{P} \neq \emptyset$ and let $P$ satisfy (4.1). Then the following condition (UPL) holds: there are $C_{i}>0$ and $j_{0}$ such that for any $v \in \mathcal{P} \mathcal{S H}\left(V_{P} \cap U_{j_{0}}\right)$, if

$$
v(x) \leq 0 \quad \text { for } x \in X_{P}, \quad v(z) \leq 1 \text { for } z \in V_{P} \cap U_{j_{0}}
$$

then

$$
v(z) \leq C_{1}|\Im(z)| \quad \text { for } z \in V_{P} \cap U_{C_{2}} .
$$

Proof. Let $j_{0}:=1$ and $C_{2}:=4 D$ for $D$ as in (4.1). Let $z \in V_{P} \cap U_{4 D}$. Choose $n \in \mathbb{N}$ such that $1 /(4 D(n+1)) \leq|\Im(z)|<1 /(4 D n)$. Since \|\|$v \mid \|_{X_{P}} \leq$ 0 and \|\|$v \|_{1} \leq 1$ by assumption, from (4.1) we get

$$
v(z) \leq\left\|\left|v\left\|_{4 D n j} \leq\right\|\right|\left|v \|_{j} / n \leq 1 / n \leq 8 D\right| \Im(z) \mid .\right.
$$

This shows (4.13) for $C_{1}:=8 D$. 
Recall that $V_{P}$ satisfies $(\mathrm{PL})_{\text {loc }}(a)$ at $a \in X_{P}$ (the local PhragménLindelöf condition at $a$ ) if there are $0<r_{2}<r_{1}$ and $A>0$ such that for any $v \in \mathcal{P} \mathcal{S H}\left(V_{P} \cap B_{r_{1}}(a)\right)$, if

$$
v(x) \leq 0 \text { for } x \in X_{P} \cap B_{r_{1}}(a), \quad v(z) \leq 1 \text { for } z \in V_{P} \cap B_{r_{1}}(a),
$$
then

$$
v(z) \leq A|\Im(z)| \quad \text { for } z \in V_{P} \cap B_{r_{2}}(a) .
$$

The uniform Phragmén-Lindelöf condition (UPL) from 4.2 can be translated into a uniform $(\mathrm{PL})_{\text {loc}}$-condition:

Proposition 4.3. Let $X_{P} \neq \emptyset$ and let $P$ satisfy (UPL). Then the following condition (UPL) loc holds: there are $r_{1}>r_{2}>0$ and $C$ such that for any $a \in X_{P}$, if $v \in \mathcal{P} \mathcal{S H}\left(V_{P} \cap B_{r_{1}}(a)\right)$ satisfies

$$
v(x) \leq 0 \text { for } x \in X_{P} \cap B_{r_{1}}(a), \quad v(z) \leq 1 \text { for } z \in V_{P} \cap B_{r_{1}}(a),
$$
then

$$
v(z) \leq C|\Im(z)| \quad \text { for } z \in V_{P} \cap B_{r_{2}}(a) .
$$

Proof. Let $r_{1}:=2 / j_{0}$ and $r_{2} \leq r_{1} / 8=1 /\left(4 j_{0}\right)$ and fix $z_{0} \in V_{P}$ with $\left|z_{0}-a\right|<r_{2}$. For $z \in V_{P} \cap U_{j_{0}}$ set

$$
u(z):=\max \left\{0, v(z)+4\left(-\left|\Re\left(z-z_{0}\right)\right|^{2}+|\Im(z)|^{2}\right) / r_{1}^{2}\right\} / 2
$$

if $\left|\Re\left(z-z_{0}\right)\right|<r_{1} / \sqrt{2}$ and $u(z)=0$ if $\left|\Re\left(z-z_{0}\right)\right| \geq r_{1} / \sqrt{2}$. Then $u$ is well-defined since $|z-a|<r_{1}$ if $\left|\Re\left(z_{0}\right)-a\right|<r_{2} \leq r_{1} / 8,\left|\Re\left(z-z_{0}\right)\right|<r_{1} / \sqrt{2}$ and $z \in U_{j_{0}}$. Furthermore, $u \in \mathcal{P} \mathcal{S H}\left(V_{P} \cap U_{j_{0}}\right)$ since

$$
4\left(-\left|\Re\left(z-z_{0}\right)\right|^{2}+|\Im(z)|^{2}\right) / r_{1}^{2}<-1 \quad \text { if }\left|\Re\left(z-z_{0}\right)\right|=r_{1} / \sqrt{2} \text { and } z \in U_{j_{0}} \text {. }
$$

Also observe that $u$ satisfies (4.12) by (4.16). Hence,

$$
v\left(z_{0}\right) \leq 2 u\left(z_{0}\right) \leq 2 C_{1}\left|\Im\left(z_{0}\right)\right| \quad \text { if } z_{0} \in U_{C_{2}} .
$$

The claim is proved for $r_{2}:=1 / \max \left\{4 j_{0}, C_{2}\right\}$.

For $l, k \in \mathbb{N}$ and $a \in \mathbb{C}^{d}$ let $b_{l, k}(a):=\left\{z \in \mathbb{C}^{d}|| \Re(z-a)|<1 / l,| \Im(z-a) \mid<1 / k\right\}$ and $b_{l, k}:=b_{l, k}(0)$. For a bounded $u \in \mathcal{P S H}\left(V_{P} \cap b_{l, k}(a)\right)$ we set, as before,

$$
\|\| u \mid\left\|_{b_{l, k}(a)}:=\sup _{z \in V_{P} \cap b_{l, k}(a)} u(z), \quad\right\| u \|_{X_{P}, B_{1 / l}(a)}:=\sup _{x \in X_{P} \cap B_{1 / l}(a)} u(x) .
$$

It is interesting to notice that $(\mathrm{PL})_{\text {loc }}(0)$ may be formulated in the spirit of (4.1), i.e. as a dual $(\bar{\Omega})_{t}$-type condition:

Proposition 4.4. The following are equivalent:

(a) $P$ satisfies $(\mathrm{PL})_{\text {loc }}(0)$ with constants $1 \geq r_{1}>r_{2}>0$ and $A$.

(b) Let $l \geq 1 / r_{2}, j \in \mathbb{N}$ and $a \in \mathbb{R}^{d} \cap B_{r_{2} / 2}(0)$. Let $u \in \mathcal{P S H}\left(V_{P} \cap b_{l, j l}(a)\right)$ be bounded above. If 
(4.18) $u(x) \leq 0$ for $x \in X_{P} \cap B_{1 / l}(a), \quad u(z) \leq 1 \quad$ for $z \in V_{P} \cap b_{l, j l}(a)$, then

$$
u(z) \leq 8 r_{1} A j l|\Im(z)| \quad \text { for } z \in V_{P} \cap b_{2 l, 8 j l}(a) .
$$

(c) There is $D \geq 1$ such that for any $l \geq 1 / r_{2}$, any $j, n \in \mathbb{N}$ and $a \in$ $\mathbb{R}^{d} \cap B_{r_{2} / 2}(0)$ and $u \in \mathcal{P} \mathcal{S H}\left(V_{P} \cap b_{l, j l}(a)\right)$ bounded above,

$$
\|v\|_{b_{2 l, D j l n}(a)} \leq\left(1-\frac{1}{n}\right)\|\| v\left\|_{X_{P}, B_{1 / l}(a)}+\frac{1}{n}\right\| v \|_{b_{l, j l}(a)} .
$$

(d) There are $R_{2}>R_{1}>0$ and $D$ such that for any $j, n$ and $k$ there is $C_{1}$ such that for any $f \in \mathcal{H}\left(B_{1 / R_{1}}(0)\right)$,

$$
\left\|f \left|\left\|_{b_{R_{2}, D j n}(0)} \leq C_{1}\left|\left\|f\left|\left\|_{b_{R_{1}, k}(0)}^{1-1 / n}\right\| f\right|\right\|_{b_{R_{1}, j}(0)}^{1-1 / n} .\right.\right.\right.\right.
$$

Proof. $(\mathrm{a}) \Rightarrow(\mathrm{b})$. Let $u \in \mathcal{P} \mathcal{S H}\left(V_{P} \cap b_{2 l, j l}(a)\right), a \in \mathbb{R}^{d} \cap B_{r_{2} / 2}(0)$, satisfy $(4.22)$

$$
u(x) \leq 0 \text { if } x \in X_{P} \cap B_{1 /(2 l)}(a), \quad u(z) \leq 1 \text { if } z \in V_{P} \cap b_{2 l, j l}(a) .
$$

Set $v(z):=\max \left\{0, u(z)+8 l^{2}\left(-|\Re(z-a)|^{2}+|\Im(z)|^{2}\right)\right\} /(8 j l)$ if $z \in V_{P} \cap$ $b_{2 l, 4 j l}(a)$ and $v(z):=0$ if $z \in\left(V_{P} \cap U_{4 j l}\right) \backslash b_{2 l, 4 j l}(a)$. Then $v \in \mathcal{P} \mathcal{S H}\left(V_{P} \cap U_{j l}\right)$ since

$$
u(z)+8 l^{2}\left(-|\Re(z-a)|^{2}+|\Im(z)|^{2}\right)<1+8 l^{2}\left(-1 /(2 l)^{2}+1 /(4 j l)^{2}\right) \leq-1 / 2
$$

by (4.22) if $z \in V_{P},|\Re(z-a)|=1 /(2 l)$ and $|\Im(z)|<1 /(4 j l)$. A similar calculation shows that $v(z) \leq 3 /(16 j l)$ if $z \in V_{P} \cap U_{4 j l}$. Hence we may define $\widetilde{v} \in \mathcal{P} \mathcal{S H}\left(V_{P}\right)$ by $\widetilde{v}(z):=\max \{v(z),|\Im(z)|\}$ if $z \in V_{P} \cap U_{4 j l}$ and $\widetilde{v}(z):=|\Im(z)|$ if $z \in V_{P} \backslash U_{4 j l}$. Clearly,

$$
\widetilde{v}(x) \leq 0 \text { if } x \in X_{P}, \quad \widetilde{v}(z) \leq \max \left\{r_{1}, 3 /(16 j l)\right\}=r_{1} \text { if } z \in V_{P} \cap B_{r_{1}}(0) \text {. }
$$

Let $z:=a+i t$ and $|t|<1 /(8 j l)$. Then $z \in B_{r_{2}}(0)$ since $j l \geq 1 / r_{2}$ and $a \in B_{r_{2} / 2}(0) \cap \mathbb{R}^{d}$, and by $(\mathrm{PL})_{\text {loc }}(0)$ we get

$$
u(a+i t) /(8 j l) \leq \widetilde{v}(a+i t) \leq r_{1} A|t| .
$$

If $u \in \mathcal{P S H}\left(V_{P} \cap b_{l, j l}(a)\right)$ satisfies (4.18) then (4.22) holds for $u(\xi+\cdot)$ instead of $u$ if $\xi \in \mathbb{R}^{d}$ and $|\xi|<1 /(2 l)$. Then (4.23) shows (4.19).

$(\mathrm{b}) \Rightarrow(\mathrm{c})$. If \|\|$v\left\|_{X_{P}, B_{1 / l}(a)}=\right\|\|v\|_{b_{l, j l}(a)}$ then (4.20) clearly holds. Let $\|v\|_{X_{P}, B_{1 / l}(a)}<\|\| v \|_{b_{l, j l}(a)}$ and set

$$
u(z):=\left(v(z)-\|v\|_{X_{P}, B_{1 / l}(a)}\right) /\left(\left.\left\|\left|v\left\|_{b_{l, j l}(a)}-\right\| v\right|\right\|\right|_{X_{P}, B_{1 / l}(a)}\right) .
$$

Then $u$ satisfies (4.18) and we get by (4.19), for $z \in b_{2 l, D j l n}(a) \subset b_{2 l, 8 j l}(a)$,

$$
u(z) \leq 8 r_{1} A l j|\Im(z)| \leq 1 / n
$$

for $D:=8 \max \left\{1, r_{1} A\right\}$. This shows (4.20).

$(\mathrm{c}) \Rightarrow(\mathrm{a})$. This follows similarly to 4.2 ( set $a=0, j=1$ and $\left.l=1 / r_{2}\right)$. 
(c) $\Rightarrow(\mathrm{d})$. We apply (4.20) for $l:=1 / r_{2}, a=0$ and $v:=\ln |f|$ to show (4.21) for $j \geq j_{0}:=1 / r_{2}$. Then (4.21) easily follows for any $j$.

$(d) \Rightarrow(c)$. This may be proved as in 4.1 , using the extension procedure from 4.3 .

Formula (4.20) is used in Section 5 to transfer (UPL) loc from $P$ to the localizations of $P$.

$(\mathrm{PL})_{\mathrm{loc}}(0)$ also implies a local version of the distance condition (2.19) and a locally uniform radial Phragmén-Lindelöf condition. Recall that the condition $(\mathrm{RPL})_{\text {loc }}(a)$ (the radial Phragmén-Lindelöf condition at $a \in X_{P}$ ) holds iff there are $0<r_{2}<r_{1}$ and $A>0$ such that for any $v \in \mathcal{P S H}\left(V_{P} \cap\right.$ $\left.B_{r_{1}}(a)\right)$, if

$$
v(x) \leq 0 \text { for } x \in X_{P} \cap B_{r_{1}}(a), \quad v(z) \leq 1 \text { for } z \in V_{P} \cap B_{r_{1}}(a),
$$

then

$$
v(z) \leq A|z-a| \quad \text { for } z \in V_{P} \cap B_{r_{2}}(a) .
$$

Condition $(\mathrm{RPL})_{\text {loc }}(a)$ was introduced in $[24,2.3]$ and has been intensively studied since then. We now get the following characterization:

Proposition 4.5. Let $0 \in X_{P}$. The following are equivalent:

(a) $V_{P}$ satisfies $(\mathrm{PL})_{\mathrm{loc}}(0)$.

(b) There are $0<r_{2}<r_{1}$ and $A>0$ such that for any $v \in \mathcal{P S H}\left(V_{P} \cap\right.$ $\left.B_{r_{1}}(0)\right)$, if

$$
v(x) \leq 0 \quad \text { for } x \in X_{P} \cap B_{r_{1}}(0), \quad v(z) \leq 1 \text { for } z \in V_{P} \cap B_{r_{1}}(0),
$$

then

$$
v(z) \leq A d\left(z, X_{P} \cap B_{r_{2}}(0)\right) \quad \text { for } z \in V_{P} \cap B_{r_{2}}(0) .
$$

Moreover,

$$
d\left(z, X_{P} \cap B_{r_{1}}(0)\right) \leq A|\Im(z)| \quad \text { for } z \in V_{P} \cap B_{r_{2}}(0) .
$$

(c) There are $0<r_{2}<r_{1}$ and $A>0$ such that $P$ satisfies (4.28) and $(\mathrm{RPL})_{\mathrm{loc}}(a)$ at any $a \in X_{P} \cap B_{r_{2}}(0)$ for these constants.

Proof. (a) $\Rightarrow$ (b). The local distance condition (4.28) is proved as in 2.7 with (4.20) (for $v(z):=-\left|\Re\left(z-z_{0}\right)\right|^{2}+\left|\Im\left(z-z_{0}\right)\right|^{2}$ and $\left.l:=1 / r_{2}\right)$ instead of (2.14). Inequality (4.27) follows from (4.15) since

$$
|\Im(z)| \leq \inf _{x \in \mathbb{R}^{d}}|z-x| \leq \inf _{x \in X_{P} \cap B_{r_{2}}(0)}|z-x|=d\left(z, X_{P} \cap B_{r_{2}}(0)\right) .
$$

(b) $\Rightarrow$ (c). With $r_{j}$ from (b) and $a \in X_{P} \cap B_{r_{2} / 2}(0)$ assume that $v$ satisfies (4.24) for $2 r_{1}$ instead of $r_{1}$. Then (4.26) holds for $v$, and (4.27) implies that for any $a \in X_{P} \cap B_{r_{2} / 2}(0)$, $v(z) \leq A d\left(z, X_{P} \cap B_{r_{2}}(0)\right) \leq A|z-a| \quad$ if $z \in V_{P} \cap B_{r_{2} / 2}(a) \subset V_{P} \cap B_{r_{2}}(0)$. 
(c) $\Rightarrow(\mathrm{b})$. As above we get (4.27) since $\inf _{a \in X_{P} \cap B_{r_{2} / 2}(0)}|z-a|=d\left(z, X_{P} \cap\right.$ $\left.B_{r_{2} / 2}(0)\right)$.

(b) $\Rightarrow(\mathrm{a})$. This is evident since

$$
d\left(z, X_{P} \cap B_{r_{1}}(0)\right) \geq d\left(z, X_{P} \cap B_{r_{2}}(0)\right) \quad \text { if } z \in B_{r_{2} / 2}(0) .
$$

Indeed, if $z \in B_{r_{2} / 2}(0)$ and $x \in B_{r_{1}}(0) \backslash B_{r_{2}}(0)$ then

$$
|z-x| \geq r_{2} / 2 \geq|z| \geq d\left(z, X_{P} \cap B_{r_{2}}(0)\right)
$$

and therefore $d\left(z, X_{P} \cap\left(B_{r_{1}}(0) \backslash B_{r_{2}}(0)\right)\right) \geq d\left(z, X_{P} \cap B_{r_{2}}(0)\right)$.

We can now add the Phragmén-Lindelöf conditions to the characterization from 3.4:

TheOREM 4.6. Let $X_{P} \neq \emptyset$. The following are equivalent:

(a) $P(D)$ admits a right inverse in $P_{*}\left(\mathbb{R}^{d}\right)_{\mathrm{b}}^{\prime}$.

(b) $V_{P}$ satisfies (4.1).

(c) $V_{P}$ satisfies (UPL).

(d) $V_{P}$ satisfies (UPL) loc and for any $C \geq 1$ there is $C_{1}$ such that for any $\alpha \in \mathbb{N}_{0}^{d}$,

$$
\left|P^{(\alpha)}(x)\right| \leq C_{1}^{|\alpha|+1}|P(x)| \quad \text { if } x \in \mathbb{R}^{d} \text { and } d\left(x, X_{P}\right) \geq 1 / C \text {. }
$$

Proof. $(\mathrm{a}) \Rightarrow(\mathrm{b}) \Rightarrow(\mathrm{c})$. This has been shown in 3.4 and 4.1 , and in 4.2 , respectively.

(c) $\Rightarrow(\mathrm{b})$. (UPL) implies that there is $C_{1}>0$ such that for any $j$ and any $v \in \mathcal{P} \mathcal{S H}\left(V_{P} \cap U_{j}\right)$, if

$$
v(x) \leq 0 \text { for } x \in X_{P}, \quad v(z) \leq 1 \text { for } z \in V_{P} \cap U_{j},
$$

then

$$
v(z) \leq C_{1} j|\Im(z)| \quad \text { for } z \in V_{P} \cap U_{C_{1} j} .
$$

To prove this, let $v$ satisfy (4.30) and set $\widetilde{v}(z):=\max \{v(z), 2 j|\Im(z)|\}$ if $z \in V_{P} \cap U_{j}$ and $\widetilde{v}(z):=2 j|\Im(z)|$ if $z \in V_{P} \cap\left(U_{1} \backslash U_{j}\right)$. Then $\widetilde{v} \in \mathcal{P} \mathcal{S H}\left(V_{P} \cap U_{1}\right)$ and

$$
\widetilde{v}(x) \leq 0 \text { if } x \in X_{P} \text { and } \widetilde{v}(z) \leq 2 j \text { if } z \in V_{P} \cap U_{j_{0}} .
$$

Now (4.31) follows from (UPL) (i.e. from (4.13) for $\widetilde{v}$ ). (4.30) and (4.31) imply (4.1) similarly to the part "(b) $\Rightarrow(\mathrm{c})$ " in the proof of 4.4 (set $u(z):=$ $\left(v(z)-\mid\|v\| \|_{j}\right) /\left(\left|\left\|v\left|\left\|_{j}-\right\|\right||v|\right\|_{X_{P}}\right)\right.$ if $\||v|\|_{X_{P}}<\left\|\left|\|v \mid\|_{j}\right)\right.$.

(b) $\Rightarrow(\mathrm{a})$. This follows from 3.4 since (4.1) implies (2.14).

$(\mathrm{c}) \Rightarrow(\mathrm{d})$. (UPL) loc was shown in 4.3. Since (a), (b) and (c) are equivalent we may use 2.7 and 2.8 (especially (2.22)) to show (4.29).

(d) $\Rightarrow(\mathrm{b})$. We have to show (4.1) for $j \geq j_{0}$, since it then also holds for $j \leq j_{0}\left(\right.$ and $j_{0} D$ instead of $\left.D\right)$. Let $l_{0}:=1 / r_{2}$. If $z \in V_{P} \cap U_{D j n}$ and $|\Re(z)-a|<1 /\left(2 l_{0}\right)$ for some $a \in X_{P}$, then the desired estimate for $v(z)$ 
directly follows from (UPL) loc and (4.20) with $l=l_{0}$. On the other hand, if $d\left(\Re(z), X_{P}\right) \geq 1 /\left(2 l_{0}\right)$ and $z \in V_{P}$ then

$$
|\Im(z)| \geq d\left(\Re(z), V_{P}\right) \geq \varepsilon>0
$$

by (4.29) and (2.2) since $z \in V_{P}$. So (4.1) holds if $j>1 / \varepsilon:=j_{0}$.

Corollary 4.7. Let $X_{P}$ be compact. Then $P(D)$ admits a right inverse in $P_{*}\left(\mathbb{R}^{d}\right)_{\mathrm{b}}^{\prime}$ iff $V_{P}$ satisfies $(\mathrm{PL})_{\mathrm{loc}}(a)$ for any $a \in X_{P}$ and there is $C$ such that for any $\alpha \in \mathbb{N}_{0}^{d}$,

$$
\left|P^{(\alpha)}(x)\right| \leq C|P(x)| \quad \text { if } x \in \mathbb{R}^{d} \text { and }|x| \geq C .
$$

Proof. If $X_{P}=\emptyset$ the statement follows from 2.6 and (2.3). Let $X_{P} \neq \emptyset$.

Necessity. This follows from 4.6(d).

Sufficiency. (4.32) implies (4.29) since $X_{P}$ is compact. Moreover, $(\mathrm{UPL})_{\text {loc }}$ follows from the assumption by a compactness argument (and e.g. 4.4).

Corollary 4.8. Let $P$ be homogeneous. Then $P(D)$ admits a right inverse in $P_{*}\left(\mathbb{R}^{d}\right)_{\mathrm{b}}^{\prime}$ iff $V_{P}$ satisfies $(\mathrm{PL})_{\text {loc }}(0)$.

Proof. Necessity. This follows from $4.6(\mathrm{~d})$ since $0 \in X_{P}$.

Sufficiency. We show (UPL). Let $v \in \mathcal{P S H}\left(V_{P} \cap U_{1}\right)$ satisfy (4.12) for $j_{0}:=1$. For $j \in \mathbb{N}$ fixed let $u(z):=v(j z)$. Then $u$ satisfies (4.18) for $l:=2 / r_{2}$ and $a=0$ (since $r_{2}<1$ without loss of generality) and we know by 4.4 (b) that there is $C$ independent of $j$ such that

$$
v(j z)=u(z) \leq C|\Im(j z)| \quad \text { if }|\Re(j z)|<j r_{2} / 2 \text { and }|\Im(j z)|<r_{2} / 8 .
$$

This proves (UPL) for $v$ since $C$ is independent of $j$.

5. Localizations. For $\zeta \in \mathbb{C}^{d}$ and $t \in \mathbb{R}$ let

$$
\widetilde{P}(\zeta, t):=\left(\sum_{\alpha}\left|P^{(\alpha)}(\zeta)\right|^{2}|t|^{2|\alpha|}\right)^{1 / 2} .
$$

We will consider limits of polynomials $Q_{s}$ of the form

$$
Q_{s}(z):=P\left(\zeta_{s}+t_{s} z\right) / \widetilde{P}\left(\zeta_{s}, t_{s}\right), \quad s \in \mathbb{N},
$$

where $\zeta_{s} \in \mathbb{C}^{d}$ and $t_{s} \neq 0$. Notice that the Taylor coefficients $\left(c_{\alpha}\right)$ of $Q_{s}$ at 0 satisfy

$$
1 /(\operatorname{deg}(P)) ! \leq\left\|\left(c_{\alpha}\right)\right\|_{2} \leq 1 .
$$

Hence any sequence of polynomials of this form has a convergent subsequence. Several types of localizations of $P$ are defined in this way and we will study the question if the crucial estimates of this paper (and especially (UPL)) are inherited by such limits. 
The set $L\left(P, x_{0}\right)$ of localizations of $P$ at $x_{0} \in X_{P} \cup\{\infty\}$ is defined as follows. Let $\mathrm{Pol}_{0}$ denote the set of non-zero polynomials in $d$ variables. For $x_{0} \in X_{P}$ let

$$
\begin{aligned}
L\left(P, x_{0}\right): & =\left\{Q \in \mathrm{Pol}_{0} \mid \exists \zeta_{s} \in \mathbb{C}^{d},\left(t_{s}\right)_{s} \subset \mathbb{R} \backslash\{0\}, c \neq 0:\right. \\
& \left.\zeta_{s} \rightarrow x_{0}, t_{s} \rightarrow 0,\left|\Im\left(\zeta_{s}\right)\right|=o\left(\left|t_{s}\right|\right), P\left(\zeta_{s}+t_{s} z\right) / \widetilde{P}\left(\zeta_{s}, t_{s}\right) \rightarrow c Q(z)\right\}
\end{aligned}
$$

and let

$$
\begin{aligned}
L(P, \infty):=\left\{Q \in \operatorname{Pol}_{0} \mid \exists \zeta_{s} \in \mathbb{C}^{d},\left(t_{s}\right)_{s} \subset \mathbb{R} \backslash\{0\} \text { bounded, } c \neq 0:\right. \\
\left.\left|\zeta_{s}\right| \rightarrow \infty,\left|\Im\left(\zeta_{s}\right)\right|=o\left(\left|t_{s}\right|\right), P\left(\zeta_{s}+t_{s} z\right) / \widetilde{P}\left(\zeta_{s}, t_{s}\right) \rightarrow c Q(z)\right\} .
\end{aligned}
$$

We say that $P$ satisfies $(\mathrm{PL})_{\text {loc }}(\infty)$ (the (uniform) local Phragmén-Lindelöf condition at $\infty$ ) if there are $r_{1}>r_{2}>0$ and $C$ such that for any $a \in X_{P}$, if $|a| \geq 1 / r_{2}, v \in \mathcal{P S H}\left(V_{P} \cap B_{r_{1}}(a)\right)$ satisfies

$$
v(x) \leq 0 \text { for } x \in X_{P} \cap B_{r_{1}}(a), \quad v(z) \leq 1 \text { for } z \in V_{P} \cap B_{r_{1}}(a)
$$

then

$$
v(z) \leq C|\Im(z)| \quad \text { for } z \in V_{P} \cap B_{r_{2}}(a) .
$$

The following result could also be obtained by the methods of $[4,3.5]$ (see also $[5,2.7]$ ). We present here a different argument based on the scaled $(\bar{\Omega})$-type estimate in $4.4(\mathrm{c})$ since it is essentially elementary and might be interesting in its own right.

Theorem 5.1. Let $P$ satisfy $(\mathrm{PL})_{\text {loc }}\left(x_{0}\right)$ for some $x_{0} \in X_{P} \cup\{\infty\}$. Then for any $Q \in L\left(P, x_{0}\right)$ defined by $\zeta_{s}, t_{s}$ as above there is $\widetilde{D}$ depending only on $\limsup \left|t_{s}\right|$ such that for any $j, n$ and $k$ there is $C_{1}$ such that for any $f \in \mathcal{H}\left(U_{j}\right)$,

$$
\|f\|_{V_{Q}, \widetilde{D} n j} \leq C_{1}\|f\|_{V_{Q}, k}^{1-1 / n}\|f\|_{V_{Q}, j}^{1 / n} .
$$

Hence (UPL) and (UPL) loc are satisfied with a constant which is uniform for any $Q \in L\left(P, x_{0}\right)$ if $x_{0} \in X_{P}$.

Proof. By a real shift of the variables we may assume that $x_{0} \in\{0, \infty\}$. (a) For $\zeta_{s}:=y_{s}+i \eta_{s}, t_{s}$ and $c \neq 0$ as above let

$$
Q_{s}(z):=P\left(\zeta_{s}+t_{s} z\right) / \widetilde{P}\left(\zeta_{s}, t_{s}\right) \rightarrow c Q(z) .
$$

For simplicity of notation we assume that $x_{1}$ is non-characteristic for $Q$. Then for $m:=\operatorname{deg}(Q)$ we have

$$
Q_{s}(z)=\sum_{J} a_{J, s}\left(z^{\prime}\right) z_{1}^{J}, \quad Q(z)=\sum_{J=1}^{m} a_{J}\left(z^{\prime}\right) z_{1}^{J}
$$

with $a_{m} \equiv$ const, where the polynomials $a_{J, s}$ converge locally uniformly to $a_{J}$ for any $J$. For $\varepsilon>0$ let

$$
V_{\varepsilon}\left(z^{\prime}\right):=V_{Q\left(z^{\prime}, \cdot\right)}+B_{\varepsilon}(0) \subset \mathbb{C} .
$$


Let $z_{0} \in V_{Q} \cap U_{\widetilde{D} n j}$ for $\widetilde{D}:=4 D C_{1} / r_{2}$, where $D$ is the constant from (4.20) and $C_{1}:=\lim \sup \left|t_{s}\right|+1$. By Hurwitz' theorem we get $s_{0}$ such that for $s \geq s_{0}$ and $\left|z^{\prime}-z_{0}^{\prime}\right| \leq 2$ each component of $V_{\varepsilon}\left(z^{\prime}\right)$ contains the same number of zeros of $Q_{s}\left(z^{\prime}, \cdot\right)$ and of $Q\left(z^{\prime}, \cdot\right)$, and the remaining zeros of $Q_{s}\left(z^{\prime}, \cdot\right)$ are larger than 3 in modulus.

(b) Let $f \in H\left(U_{j}\right)$ be such that $\|f\|_{V_{Q}, j}<\infty$. By 2.3 we can assume that $f \in P_{*, j}$. Let $0<\varepsilon<r_{2} /\left(4 C_{1} k n D\right)$ be fixed. We then find $\widetilde{z}$ with $\left|\widetilde{z}-z_{0}\right|<\varepsilon$ and $Q_{s}(\widetilde{z})=0$ by (a), that is, $P\left(\xi_{s}\right)=0$ for $\xi_{s}:=\zeta_{s}+t_{s} \widetilde{z}$. Let $x_{s}:=\Re\left(\xi_{s}\right)$. Then $x_{s} \in B_{r_{2} / 2}(0)$ for large $s$ if $x_{0}=0$ (and $\left|x_{s}\right| \geq 1 / r_{2}$ for large $s$ if $x_{0}=\infty$ ). We may thus apply 4.4 for $a:=x_{s}$ for large $s$. Let $l:=C_{1} /\left(r_{2}\left|t_{s}\right|\right)$. Then $l \geq 1 / r_{2} \geq 1$ and $\xi_{s} \in b_{2 l, D j l n}\left(x_{s}\right)$ for large $s$ by the definition of $l$ since $z_{0} \in U_{\widetilde{D} n j}, \varepsilon<r_{2} /\left(4 C_{1} k n D\right) \leq r_{2} /\left(4 C_{1} j n D\right)$ and $\left|\eta_{s}\right|=\left|\Im\left(\zeta_{s}\right)\right|=o\left(\left|t_{s}\right|\right)$. Let $\widetilde{f}(z):=f\left(\left(z-\zeta_{s}\right) / t_{s}\right)$. Then $f(\widetilde{z})=\widetilde{f}\left(\xi_{s}\right)$, and formula (4.20) in 4.4 implies that

$$
\left|f\left(z_{0}\right)\right| e^{\left|z_{0}\right| /(\widetilde{D} n j)} \leq\left(\varepsilon \sup _{z \in U_{2 j}}\left|f^{\prime}(z)\right|+\left|\widetilde{f}\left(\xi_{s}\right)\right|\right) e^{\left|z_{0}\right| /(n j)}
$$

$$
\leq \varepsilon e^{\left|z_{0}\right| /(n j)} \sup _{z \in U_{2 j}}\left|f^{\prime}(z)\right|+\left(e^{\left|z_{0}\right| / k}|| \widetilde{f} \mid \|_{X_{P}, B_{1 / l}\left(x_{s}\right)}\right)^{1-1 / n}\left(e^{\left|z_{0}\right| / j}\|\tilde{f} \mid\|_{b_{l, j l}\left(x_{s}\right)}\right)^{1 / n}
$$

since $l \geq 1 / r_{2} \geq 1$. If $x \in X_{P}$ and $\left|x_{s}-x\right| \leq 1 / l$ then $Q_{s}(\widetilde{x})=0$ for $\widetilde{x}:=\left(x-\zeta_{s}\right) / t_{s}$ and $\left|\widetilde{x}-z_{0}\right| \leq 2$ for large $s$ by the definition of $l$ and $x_{s}$. By the remarks in (a) we thus find $\widehat{z} \in V_{Q}$ with $|\widehat{z}-\widetilde{x}|<\varepsilon \leq 1 /(4 k)$ and therefore

$$
e^{\left|z_{0}\right| / k}\left\|\widetilde { f } \left|\left\|_{X_{P}, B_{1 / l}\left(x_{s}\right)} \leq \varepsilon e^{\left|z_{0}\right| / k} \sup _{z \in U_{2 k}}\left|f^{\prime}(z)\right|+C_{2}\right\| f \|_{V_{Q}, k}\right.\right.
$$

for large $s$ since $l \geq 1$ and $\widetilde{x} \in U_{4 k}\left(\right.$ as $\left.\left|\eta_{s}\right|=o\left(\left|t_{s}\right|\right)\right)$. Similarly,

$$
e^{\left|z_{0}\right| / j}\left\|\tilde { f } \left|\left\|_{b_{l, j l}\left(x_{s}\right)} \leq \varepsilon e^{\left|z_{0}\right| / j} \sup _{z \in U_{2 j}}\left|f^{\prime}(z)\right|+C_{2}\right\| f \|_{V_{Q}, j} .\right.\right.
$$

Now (5.1) follows if we insert these estimates in (5.2) and let $\varepsilon \downarrow 0$. The last claim of the theorem follows from 3.4 and 4.6 since $\lim \sup \left|t_{s}\right|=0$ by the definition of $L\left(P, x_{0}\right)$ for $x_{0} \in X_{P}$.

COROLlaRY 5.2. The operator $Q(D)$ admits a right inverse in $P_{*}\left(\mathbb{R}^{d}\right)_{\mathrm{b}}^{\prime}$ for any $Q \in L\left(P, x_{0}\right)$ and $x_{0} \in X_{P} \cup \infty$ if $P(D)$ admits a right inverse in $P_{*}\left(\mathbb{R}^{d}\right)_{\mathrm{b}}^{\prime}$.

The following example shows that the uniformity of the constants in $(\mathrm{PL})_{\text {loc }}(\infty)$ is important, that is, we may not replace $(\mathrm{PL})_{\text {loc }}(\infty)$ in 5.1 by the assumption that $(\mathrm{PL})_{\text {loc }}(a)$ holds for any $a \in X_{P}$ if $|a|$ is large.

EXAMPLE 5.3. Let $P(x):=x_{3}\left(x_{1}^{2} x_{2}^{2}+1\right)-1$. Then $X_{P}$ consists of regular points and therefore $P$ satisfies $(\mathrm{PL})_{\text {loc }}\left(x_{0}\right)$ for any $x_{0} \in X_{P}$. However, 
$Q(x):=x_{1}^{2} x_{2}^{2}+1 \in L(P, \infty)$ (use $\zeta_{s}:=(0,0, s)$ and $\left.t_{s}:=1, s \in \mathbb{N}\right)$ and $Q$ does not satisfy (5.1) since $Q(D)$ does not admit a right inverse in $P_{*}\left(\mathbb{R}^{d}\right)^{\prime}$ by 2.6. Indeed, $X_{Q}=\emptyset$ while $z_{j}:=(j, i / j, 0) \in V_{Q}$ and $\Im\left(z_{j}\right) \rightarrow 0$.

Also, the principal part $P_{m}$ of $P$ is a limit of the general form indicated at the beginning of this section, since $c P_{m}(x)=\lim _{s \rightarrow \infty} P(s x) / \widetilde{P}(0, s)$. However, we get a negative result in this case since the existence of a right inverse for $P(D)$ in $P_{*}\left(\mathbb{R}^{d}\right)_{\mathrm{b}}^{\prime}$ in general is not inherited by the principal part $P_{m}(D)$ :

EXAMPLE 5.4. Let $P(x):=\sum_{j=1}^{d} x_{j}^{2}+1$. Then $P(D)$ admits a right inverse in $P_{*}\left(\mathbb{R}^{d}\right)_{\mathrm{b}}^{\prime}$ by 2.1 while the principal part (i.e. the Laplacian) does not.

Proof. Observe that $P_{2}(x)=\sum_{j=1}^{d} x_{j}^{2}$ does not satisfy (PL) loc $(0)$ by [22]. Hence the claim follows from 4.6.

\section{References}

[1] J. Bonet, C. Fernández and R. Meise, Operators of solution for convolution equations, Note Mat. 17 (1997), 1-12.

[2] R. W. Braun, Hörmander's Phragmén-Lindelöf principle and irreducible singularities of codimension 1, Boll. Un. Mat. Ital. A (7) 6 (1992), 339-348.

[3] - , The surjectivity of a constant coefficient homogeneous differential operator on the real analytic functions and the geometry of its symbol, Ann. Inst. Fourier (Grenoble) 45 (1995), 223-249.

[4] R. W. Braun, R. Meise and B. A. Taylor, The geometry of analytic varieties satisfying the local Phragmén-Lindelöf condition and a geometric characterization of partial differential operators that are surjective on $\mathcal{A}\left(\mathbb{R}^{4}\right)$, Trans. Amer. Math. Soc. 356 (2004), 1315-1383.

[5] - - - - , Nearly hyperbolic varieties and Phragmén-Lindelöf conditions, in: I. Sabadini (ed.), Harmonic Analysis, Signal Processing and Complexity, Progr. Math. 238, Birkhäuser, Boston, 2005, 81-95.

[6] I. M. Gelfand and G. E. Shilov, Generalized Functions, Vol. 2, Academic Press, New York, 1968.

[7] L. Hörmander, On the existence of real analytic solutions of partial differential operators with constant coefficients, Invent. Math. 21 (1973), 151-182.

[8] - , The Analysis of Linear Partial Differential Operators, I, II, Springer, Berlin, 1983.

[9] A. Kaneko, Introduction to Hyperfunctions, Kluwer, Dordrecht, 1988.

[10] T. Kawai, On the theory of Fourier hyperfunctions and its applications to partial differential equations with constant coefficients, J. Fac. Sci. Univ. Tokyo Sect. I A Math. 17 (1970), 467-517.

[11] M. Langenbruch, Solution operators for partial differential equations in weighted Gevrey spaces, Michigan Math. J. 37 (1990), 3-24. 
[12] M. Langenbruch, Real roots of polynomials and right inverses for partial differential operators in the space of tempered distributions, Proc. Roy. Soc. Edinburgh Sect. A 114 (1990), 169-179.

[13] - Splitting of the $\bar{\partial}$-complex in weighted spaces of square integrable functions, Rev. Mat. Univ. Complut. Madrid 5 (1992), 201-223.

[14] - Continuous linear right inverses for convolution operators in spaces of real analytic functions, Studia Math. 110 (1994), 65-82.

[15] - Hyperfunction fundamental solutions of surjective convolution operators on real analytic functions, J. Funct. Anal. 131 (1995), 78-93.

[16] - Hermite functions and weighted spaces of generalized functions, Manuscripta Math. 119 (2006), 269-285.

[17] —, Division problems for Fourier ultrahyperfunctions, Bull. Belg. Math. Soc. Simon Stevin 14 (2007), to appear.

[18] M. Langenbruch, Generalized Fourier expansion in kernels of convolution operators on Fourier hyperfunctions, Analysis 27 (2007), 1001-1023.

[19] - Convolution operators on Fourier hyperfunctions, preprint.

[20] M. Langenbruch and S. Momm, Complemented submodules in weighted spaces of analytic functions, Math. Nachr. 157 (1992), 263-276.

[21] R. Meise and B. A. Taylor, Splitting of closed ideals in (DFN)-algebras of entire functions and the property (DN), Amer. Math. Soc. Transl. 302 (1987), 341-370.

[22] R. Meise, B. A. Taylor and D. Vogt, Characterization of the linear partial differential operators with constant coefficients that admit a continuous linear right inverse, Ann. Inst. Fourier (Grenoble) 40 (1990), 619-655.

[23] - , 一, - Equivalence of analytic and plurisubharmonic Phragmén-Lindelöf conditions, in: Proc. Sympos. Pure Math. 52, Part 3, Amer. Math. Soc., Providence, RI, 1991, 287-308.

[24] - , - - Extremal plurisubharmonic functions of linear growth on algebraic varieties, Math. Z. 219 (1995), 515-537.

[25] - , - - - Continuous linear right inverses for partial differential operators on nonquasianalytic classes and on ultradistributions, Math. Nachr. 180 (1996), 213-242.

[26] - - - - , Phragmén-Lindelöf principles on algebraic varieties, J. Amer. Math. Soc. 11 (1998), 1-39.

[27] R. Meise and D. Vogt, Introduction to Functional Analysis, Clarendon Press, Oxford, 1997.

[28] S. N. Melikhov and S. Momm, Solution operators for convolution equations on germs of analytic functions on compact convex sets in $\mathbb{C}^{N}$, Studia Math. 117 (1995), 79-99.

[29] M. Poppenberg and D. Vogt, A tame splitting theorem for exact sequences of Fréchet spaces, Math. Z. 219 (1995), 141-161.

[30] M. Sato, Theory of hyperfunctions, Sûgaku 10 (1958), 1-27 (in Japanese).

[31] D. Vogt, Eine Charakterisierung der Potenzreihenräume von endlichem Typ und ihre Folgerungen, Manuscripta Math. 37 (1982), 269-301.

[32] - Extension operators for real analytic functions on compact subvarieties of $\mathbb{R}^{d}$, J. Reine Angew. Math. 606 (2007), 217-233.

[33] M.-J. Wagner, Quotientenräume von stabilen Potenzreihenräumen endlichen Typs, Manuscripta Math. 31 (1980), 97-109.

[34] G. Zampieri, Propagation of singularity and existence of real analytic solutions of locally hyperbolic equations, J. Fac. Sci. Univ. Tokyo Sect. I A Math. 31 (1984), 373-390. 
[35] G. Zampieri, An application of the fundamental principle of Ehrenpreis to the existence of global Gevrey solutions of linear partial differential equations, Boll. Un. Mat. Ital. B (6) 5 (1986), 361-392.

Department of Mathematics

University of Oldenburg

D-26111 Oldenburg, Germany

E-mail: Michael.Langenbruch@uni-oldenburg.de

Received January 23, 2007

Revised version August 8, 2007

(6091) 